\title{
SUPERSINGULAR K3 SURFACES ARE UNIRATIONAL
}

\author{
CHRISTIAN LIEDTKE
}

\begin{abstract}
We show that supersingular K3 surfaces in characteristic $p \geq 5$ are related by purely inseparable isogenies. This implies that they are unirational, which proves conjectures of Artin, Rudakov, Shafarevich, and Shioda. As a byproduct, we exhibit the moduli space of rigidified K3 crystals as an iterated $\mathbb{P}^{1}$-bundle over $\mathbb{F}_{p^{2}}$. To complete the picture, we also establish Shioda-Inose type isogeny theorems for K3 surfaces with Picard rank $\rho \geq 19$ in positive characteristic.
\end{abstract}

\section{INTRODUCTION}

1 The Picard rank $\rho$ of a complex K3 surface satisfies $\rho \leq 20$. In [SI77], [I78], Shioda and Inose classified complex K3 surfaces with Picard rank 20, so-called singular K3 surfaces, showed that they can be defined over numbers, and thus, form a countable set and have no moduli. They also showed that such a surface rationally dominates and is rationally dominated by a Kummer surface. This is related to a conjecture of Shafarevich [Sh71], according to which every Hodgeisogeny between the transcendental lattices of two complex K3 surfaces is induced by a rational map or a rational correspondence - we refer to Section 2.2 for details.

The first result of this article is an extension of the Shioda-Inose theorem to positive characteristic:

Theorem. Let X be a K3 surface in odd characteristic with Picard rank 19 or 20. Then, there exists an ordinary Abelian surface A and dominant, rational maps

$$
\operatorname{Km}(A) \rightarrow X \rightarrow \operatorname{Km}(A),
$$

both of which are generically finite of degree 2.

We refer to Theorem 2.6 for more precise statements, fields of definition, as well as lifting results. For example, singular K3 surfaces in odd characteristic can be defined over finite fields, and thus, also these surfaces form a countable set and have no moduli.

Artin [Ar74a] observed that there do not exist K3 surfaces with Picard rank 21 in any characteristic. On the other hand, Tate [Ta65] and Shioda [Sh77b] gave examples of K3 surfaces with Picard rank 22 in positive characteristic, so-called Shiodasupersingular K3 surfaces. Artin [Ar74a] showed that Shioda-supersingular K3

Date: April 8, 2014.

2010 Mathematics Subject Classification. 14J28, 14G17, 14M20, 14D22.

${ }^{1}$ Due to a mistake in Proposition 3.5, our results merely show that supersingular K3 surfaces are related by sequences of very special correspondences, which is not sufficient to deduce their unirationality. The unirationality conjecture thus remains a conjecture. See the appendix for erratum. 
surfaces are Artin-supersingular, that is, their formal Brauer groups are of infinite height. It follows from recent progress in the Tate-conjecture for K3 surfaces due to Charles [Ch13], Madapusi Pera [MP13], and Maulik [Mau12] that a K3 surface in odd characteristic is Artin-supersingular if and only if it is Shioda-supersingular.

Artin [Ar74a] also showed that supersingular K3 surfaces form 9-dimensional families, which is in contrast to the above mentioned rigidity of singular K3 surfaces. Moreover, Shioda [Sh77b] showed that Tate's and his examples are unirational, another property of $\mathrm{K} 3$ surfaces that can happen in positive characteristic only. Since unirational K3 surfaces are supersingular as shown by Shioda [Sh74], this led several people to conjecture the converse:

Conjecture (Artin, Rudakov, Shafarevich, Shioda). A K3 surface is supersingular if and only if it is unirational.

Shioda [Sh77b] established this conjecture for supersingular Kummer surfaces in odd characteristic, Rudakov and Sharafevich [RS78] showed it in characteristic 2 and for K3 surfaces with Artin invariant $\sigma_{0} \leq 6$ in characteristic 3, and Pho and Shimada [PS06] for K3 surfaces with Artin invariant $\sigma_{0} \leq 3$ in characteristic 5 . We refer to [Ka87] and [I-L13] for some refinements. In particular, there do exist unirational $\mathrm{K} 3$ surfaces in every positive characteristic.

The key result of this article is a structure theorem for supersingular K3 surfaces, which was posed as a question by Rudakov and Shafarevich in [RS78], and which is similar to the Shioda-Inose theorem for singular K3 surfaces.

Theorem. Let $X$ and $X^{\prime}$ be supersingular K3 surfaces in characteristic $p \geq 5$ with Artin invariants $\sigma_{0}$ and $\sigma_{0}^{\prime}$, respectively. Then, there exist dominant and rational maps

$$
X \rightarrow X^{\prime} \rightarrow X
$$

both of which are purely inseparable and generically finite of degree $p^{2 \sigma_{0}+2 \sigma_{0}^{\prime}-4}$.

In [SI77], [I78], Shioda and Inose introduced a notion of isogeny for singular K3 surfaces over the complex numbers, which was extended to other types of complex K3 surfaces by Morrison [Mo84], Mukai [Mu87], and Nikulin [Ni91]. We refer to Section 2.2 for an extension of this notion to positive characteristic, and using this terminology, our structure theorem says that all supersingular K3 surfaces are mutually purely inseparably isogenous.

Our theorem also fits into Shafarevich's conjecture [Sh71] mentioned above: supersingular K3 surfaces are precisely those K3 surfaces without transcendental cycles in their second $\ell$-adic cohomology. Thus, their "transcendental lattices" should be thought of as being zero, thus mutually isogenous, and by our theorem, they are all related by rational maps. We refer to Section 2.2 for details.

Our theorem also explains why supersingular K3 surfaces form 9-dimensional families, whereas singular K3 surface have no moduli: in both cases, these surfaces are isogenous to Kummer surfaces. For singular K3 surfaces, the isogeny is separable and does not deform. For supersingular K3 surfaces, the isogeny can be chosen purely inseparable, and deforms in families. We refer to Remark 5.2 for details. 
The main tool to proving this theorem is that a Jacobian elliptic fibration $X \rightarrow \mathbb{P}^{1}$ on a supersingular $\mathrm{K} 3$ surface with Artin invariant $\sigma_{0}$ admits a deformation that is a one-dimensional family of elliptic supersingular K3 surfaces, such that all elliptic fibrations in this family are generically torsors under $X \rightarrow \mathbb{P}^{1}$. We call this a moving torsor family and refer to Section 3.1 for details. Moreover, the generic fiber of this family has Artin invariant $\sigma_{0}+1$ and is related to the special fiber $X$ by a purely inseparable isogeny, see Theorem 3.6.

In [Og79], Ogus introduced moduli spaces $\mathscr{M}_{N}$ of $N$-rigidified K3-crystals, where $N$ is a supersingular K3 lattice. If $N$ and $N_{+}$denote supersingular K3 lattices in odd characteristic of Artin invariants $\sigma_{0}$ and $\sigma_{0}+1$, respectively, then these moving torsor families induce a structure of a $\mathbb{P}^{1}$-bundle.

Theorem. There exists a surjective morphism

$$
\mathscr{M}_{N_{+}} \rightarrow \mathscr{M}_{N}
$$

together with a section, which turns $\mathscr{M}_{N_{+}}$into a $\mathbb{P}^{1}$-bundle over $\mathscr{M}_{N}$.

Using Ogus' Torelli theorem [Og83], we use this $\mathbb{P}^{1}$-bundle structure to show that every supersingular K3 surface of Artin invariant $\sigma_{0}+1$ is purely inseparably isogenous to one of Artin invariant $\sigma_{0}$, and, by induction on the Artin invariant, we obtain our theorem. We refer to Theorem 4.3 and Theorem 4.5 for details.

As already mentioned above, Shioda $[\overline{S h 77 b}]$ proved that supersingular Kummer surfaces in odd characteristic are unirational. Combined with our structure theorem, this establishes the Artin-Rudakov-Shafarevich-Shioda conjecture.

Theorem. Supersingular K3 surfaces in characteristic $p \geq 5$ are unirational.

Together with results of Artin, Shioda, and the recent proof of the Tate-conjecture for K3 surfaces in odd characteristic, we obtain the following equivalence.

Theorem. For a $K 3$ surface $X$ in characteristic $p \geq 5$, the following conditions are equivalent:

(1) $X$ is unirational.

(2) The Picard rank of $X$ is 22.

(3) The formal Brauer group of $X$ is of infinite height.

(4) For all $i$, the $F$-crystal $H_{\text {cris }}^{i}(X / W)$ is of slope $i / 2$.

We refer to Section 3.4, Section 4.4 and Section 5.4 for partial results in small characteristics. For example, if the Rudakov-Shafarevich theorem [RS82] on potential good reduction of supersingular K3 surfaces were known to hold in characteristic 3 , then the above theorems would hold in characteristic 3 as well.

Recently, Lieblich [Li14] gave a new proof of the unirationality of supersingular K3 surfaces, following a simplified form of our strategy .

This article is organized as follows:

In Section 2, after reviewing formal Brauer groups, several notions of supersingularity, and introducing purely inseparable isogenies, we classify K3 surfaces 
with Picard ranks 19 and 20 in odd characteristic, which generalizes the classical Shioda-Inose theorem.

In Section 3, we show how a supersingular K3 surface with Artin invariant $\sigma_{0}$ together with a Jacobian elliptic fibration gives rise to a one-dimensional family of elliptic supersingular K3 surfaces that are generically torsors under this Jacobian fibration, and whose generic fiber has Artin invariant $\sigma_{0}+1$. Moreover, we show how these torsors are related to the trivial torsor by purely inseparable isogenies.

In Section 4, we interpret these one-dimensional families of torsors in terms of Ogus' moduli spaces of supersingular K3 crystals. As an interesting byproduct, we find that these moduli spaces are related to each other by iterated $\mathbb{P}^{1}$-bundles, together with a moduli interpretation of this structure. In particular, this gives a new description of these moduli spaces.

In Section 5, we use the results of the previous sections to prove that supersingular K3 surfaces are connected by purely inseparable isogenies. Since Shioda showed that supersingular Kummer surfaces are unirational, we conclude that all supersingular K3 surfaces are unirational. Finally, we also characterize unirational Enriques surfaces.

Acknowledgements. It is a pleasure for me to thank Xi Chen, Igor Dolgachev, Gerard van der Geer, Brendan Hassett, Daniel Huybrechts, Toshiyuki Katsura, Frans Oort, Matthias Schütt, Tetsuji Shioda, Burt Totaro, and the referee for discussions, comments, and pointing out inaccuracies. I especially thank Olivier Benoist and Max Lieblich for pointing out mistakes in earlier versions of this article and helping me to fix them. I gratefully acknowledge funding from DFG via Transregio SFB 45, as part of this article was written while staying at Bonn university.

\section{NON-SUPERSINGULAR K3 SURFACES WITH LARGE PICARD NUMBER}

In this section, we first review the formal Brauer group, and discuss several notions of supersingularity for K3 surfaces. Then, we classify non-supersingular K3 surfaces with large Picard rank in positive characteristic, which establishes a structure result similar to the Shioda-Inose theorem over the complex numbers.

2.1. Formal Brauer groups, supersingularity, and Picard ranks. Let $X$ be a K3 surface over a field $k$. By results of Artin and Mazur [AM77], the functor on local Artinian $k$-algebras with residue field $k$ defined by

$$
\begin{array}{cccc}
\Phi_{X / k}^{2}:(\text { Art } / k) & \rightarrow & \text { (Abelian groups }) \\
R & \mapsto \operatorname{ker}\left(H_{\text {êt }}^{2}\left(X \times_{k} \operatorname{Spec} R, \mathbb{G}_{m}\right) \rightarrow H_{\text {êt }}^{2}\left(X, \mathbb{G}_{m}\right)\right)
\end{array}
$$

is pro-representable by a one-dimensional formal group law $\widehat{\operatorname{Br}}(X)$, which is called the formal Brauer group. Over algebraically closed fields of positive characteristic, one-dimensional formal group laws are classified by their height, and Artin Ar74a, Theorem (0.1)] showed that the height $h$ of the formal Brauer group of a K3 surface satisfies $1 \leq h \leq 10$ or $h=\infty$. 
Definition 2.1. Let $X$ be a K3 surface over a field of positive characteristic and let $h$ be the height of its formal Brauer group. Then, $X$ is called ordinary if $h=1$, and $X$ is called Artin-supersingular if $h=\infty$.

The general picture is as follows: a smooth and proper variety $X$ over a perfect field of positive characteristic is called ordinary, if the Hodge- and Newtonpolygons of all its crystalline cohomology groups coincide. It is called supersingular, if the Newton-polygons of all its crystalline cohomology groups are straight lines, that is, if the $F$-crystal $H_{\text {cris }}^{i}(X / W)$ is of slope $i / 2$ for all $i$. Now, if $X$ is a $\mathrm{K} 3$ surface, this general definition translates into a condition on $H_{\text {cris }}^{2}(X / W)$ only. More precisely, being ordinary translates into having slopes $(0,1,2)$, and being supersingular into being of slope 1. By [II79, Section II.7.2], the slopes of $H_{\text {cris }}^{2}(X / W)$ in terms of the height $h$ of the formal Brauer group are $\left(1-\frac{1}{h}, 1,1+\frac{1}{h}\right)$. Thus, for K3 surfaces, Definition 2.1 coincides with the general picture.

For surfaces, Shioda [Sh74] introduced another notion of supersingularity. To explain it, we note that the first Chern class map $c_{1}: \operatorname{NS}(X) \rightarrow H^{1}\left(\Omega_{X}^{1}\right)$ is injective over the complex numbers, which implies that the Picard rank $\rho$ of a smooth complex projective variety is bounded above by $h^{1}\left(\Omega_{X}^{1}\right)$. For complex K3 surfaces, this gives the estimate $\rho \leq 20$. In positive characteristic, Igusa [Ig60] established the inequality $\rho \leq b_{2}$, which, for K3 surfaces, only gives the estimate $\rho \leq 22$. However, this bound is sharp, since Tate [Ta65] and Shioda [Sh77b] showed that there do exist K3 surfaces with Picard rank 22 in positive characteristic.

Definition 2.2. Let $X$ be a K3 surface over an algebraically closed field. Then, $X$ is called singular if $\rho=20$, and it is called Shioda-supersingular if $\rho=22$.

The relation between these two notions of supersingularity is as follows: In Ar74a, Theorem (0.1)], Artin showed that a K3 surface whose formal Brauer group is of finite height $h$ satisfies $\rho \leq b_{2}-2 h$. This implies that Shioda-supersingular K3 surfaces are Artin-supersingular. In [Ar74a, Theorem (4.3)], Artin proved that Artin-supersingular K3 surfaces that are elliptic are Shioda-supersingular. In general, the equivalence of Artin- and Shioda-supersingularity follows from the Tate-conjecture for supersingular K3 surfaces. Since this has been recently established in odd characteristic by Charles [Ch13], Madapusi Pera [MP13], and Maulik [Mau12], we can summarize these results as follows.

Theorem 2.3 (Artin, Charles, Madapusi Pera, Maulik, et al.). For a K3 surface X in odd characteristic, the following are equivalent:

(1) $X$ is Shioda-supersingular, that is, $\rho=22$.

(2) $X$ is Artin-supersingular, that is, $h(\widehat{\operatorname{Br}}(X))=\infty$.

(3) For all $i$, the $F$-crystal $H_{\text {cris }}^{i}(X / W)$ is of slope $i / 2$.

By [Ar74a, Section 4], the discriminant of the Néron-Severi lattice of a Shiodasupersingular $\mathrm{K} 3$ surface is equal to $-p^{2 \sigma_{0}}$ for some integer $1 \leq \sigma_{0} \leq 10$.

Definition 2.4. The integer $\sigma_{0}$ is called the Artin-invariant of $X$.

The Artin invariant $\sigma_{0}$ gives rise to a stratification of the moduli space of Shiodasupersingular K3 surfaces [Ar74a, Section 7], and it determines the Néron-Severi 
lattice of a Shioda-supersingular K3 surface up to isometry [RS78, Section 1]. We refer the interested reader to the overview articles by Shioda [Sh79] and RudakovShafarevich [RS81] for basic properties of Shioda-supersingular K3 surfaces, details and further references.

2.2. Isogenies between $\mathbf{K} \mathbf{3}$ surfaces. For Abelian varieties, the notion of isogeny is classical. For K3 surfaces, there are several and conflicting extensions of this notion, and we refer to [Mo87, Section 1] for an overview. Following Inose [I78], we use the most naive one, which is sufficient for the purposes of this article.

Definition 2.5. Let $X$ and $Y$ be varieties of the same dimension over a perfect field of positive characteristic $p$. An isogeny of degree $n$ from $X$ to $Y$ is a dominant, rational, and generically finite map $X \rightarrow Y$ of degree $n$. A purely inseparable isogeny of height $h$ is an isogeny that is purely inseparable of degree $p^{h}$.

For Abelian varieties $A, B$ and an isogeny $A \rightarrow B$, there exists an integer $n$ such that multiplication by $n: A \rightarrow A$ factors through this isogeny. Such a factorization gives rise to an isogeny $B \rightarrow A$, and in particular, being isogenous is an equivalence relation. Over the complex numbers, K3 surfaces with Picard rank 20 are related to Kummer surfaces by isogenies, and the existence of an isogeny in the other direction is a true, but non-trivial fact, see [SI77], [I78], and [Ma13].

Coming back to Definition 2.5, if $X \rightarrow Y$ is a purely inseparable isogeny of height $h$, the $h$-fold $k$-linear Frobenius $F^{h}: X \rightarrow X^{(p)}$ factors through this isogeny, inducing an isogeny $Y \rightarrow X^{(p)}$, which is purely inseparable of height $(d-1) h$, where $d$ is the dimension of $X$ and $Y$. Since the ground field is perfect, we can identify $X$ with $X^{(p)}$, and thus, purely inseparable isogenies define an equivalence relation.

Since it motivates some of our results later on and sheds another light on them, let us shortly discuss a conjecture of Shafarevich concerning complex K3 surfaces: let $X$ and $Y$ be complex $\mathrm{K} 3$ surfaces with transcendental lattices $T(X)$ and $T(Y)$. If $\rho(X)=\rho(Y)=20$, then $T(X)$ and $T(Y)$ are of rank 2, and the Shioda-Inose theorem [SI77] says that every isogeny $T(X) \rightarrow T(Y)$ preserving Hodge structures induces and is induced by an isogeny between the corresponding surfaces. Morrison [Mo84], Mukai [Mu87], and Nikulin [Ni87], [Ni91] generalized these results to K3 surfaces, whose transcendental lattices are of higher rank. Moreover, Shafarevich [Sh71] conjectured that every Hodge isogeny between transcendental lattices of complex K3 surfaces is induced by an isogeny, or, by a rational correspondence. Here, the right definition of isogeny for $\mathrm{K} 3$ surfaces is one difficulty, and we refer to [Mo87, Section 1] for a discussion and the relation of Shafarevich's conjecture to the Hodge conjecture. We note that results of Chen [Ch10] imply that Shafarevich's conjecture cannot be true if one only allows isogenies in the sense of our naive Definition 2.5

In positive characteristic, a K3 surface $X$ is Shioda-supersingular if and only if every class in $H_{\text {êt }}^{2}\left(X, \mathbb{Q}_{\ell}\right)$ is algebraic if and only if the cokernel of $c_{1}: \mathrm{NS}(X) \rightarrow$ $H_{\text {cris }}^{2}(X / W)$ is a $W$-module that is torsion. Therefore, the "transcendental lattices" of Shioda-supersingular K3 surfaces should be thought of as being zero, in which 
case they would all be isogenous for trivial reasons. Now, if one believes in a characteristic- $p$ version of Shafarevich's conjecture (even whose precise formulation is unclear to the author at the moment), one might expect that all Shiodasupersingular K3 surfaces are related by isogenies. This was posed as Question 8 by Rudakov and Shafarevich at the end of [RS78], and we shall prove it in Theorem 5.1 below.

2.3. The Shioda-Inose theorem in odd characteristic. In this subsection, we classify non-supersingular K3 surfaces with Picard rank $\rho \geq 19$ in odd characteristic and establish an analog of the Shioda-Inose theorem [SI77], [I78]. The idea is to show that such surfaces are ordinary, which implies that we can lift them to the Witt ring together with their Picard groups. Then, we use the Shioda-Inose theorem in characteristic zero to deduce the structure result in odd characteristic.

Theorem 2.6. Let $X$ be a K3 surface with Picard rank $19 \leq \rho \leq 21$ over an algebraically closed field $k$ of odd characteristic. Then,

(1) $X$ is an ordinary $K 3$ surface, and

(2) $X$ lifts projectively together with its Picard group to $\operatorname{Spec} W(k)$.

Moreover,

(3) If $\rho=19$, then there exists an ordinary Abelian surface A over $k$, and isogenies of degree 2

$$
\mathrm{Km}(A) \rightarrow X \rightarrow \operatorname{Km}(A) .
$$

Moreover, neither X nor A can be defined over a finite field.

(4) If $\rho=20$, then there exist two ordinary and isogenous elliptic curves $E$ and $E^{\prime}$ over $k$, and isogenies of degree 2

$$
\mathrm{Km}\left(E \times E^{\prime}\right) \rightarrow X \rightarrow \mathrm{Km}\left(E \times E^{\prime}\right) .
$$

Moreover, $X$ can be defined over a finite field. The lift of $(X, \operatorname{Pic}(X))$ is unique and coincides with the canonical Serre-Tate lift of $X$.

(5) K3 surfaces with Picard rank $\rho=21$ do not exist.

Remark 2.7. Non-existence of K3 surfaces with Picard rank 21 was already observed by Artin [Ar74a, p. 544]. Independently, Jang [Ja13, Section 4] obtained a similar classification result for K3 surfaces with Picard rank $\rho=20$.

PROOF. We proceed in several steps:

STEP 1: $X$ is ordinary and $\rho=21$ is impossible.

Let $h$ be the height of the formal Brauer group. Since $\rho<22$, we deduce $h<\infty$ from [Ar74a, Theorem 1.7]. But then, the inequalities $\rho \leq b_{2}-2 h \leq 20$ from Ar74a, Theorem 0.1] show that $\rho=21$ is impossible. They also show that $X$ is ordinary, that is, $h=1$, if $19 \leq \rho \leq 20$. This establishes claims (1) and (5).

STEP 2: There exists a projective lift of the pair $(X, \operatorname{Pic}(X))$ to $W(k)$.

Since $X$ is ordinary, there exists a canonical formal lift $\mathscr{X} \rightarrow \operatorname{Spf} W(k)$, the Serre-Tate lift. By $[\mathrm{Ny}$, Proposition 1.8], it has the property that $\operatorname{Pic}(X)$ lifts to 
$\mathscr{X}$. In particular, lifting an ample invertible sheaf, it follows from Grothendieck's existence theorem that $\mathscr{X}$ is algebraizable. This establishes claim (2).

STEP 3: If $\rho=20$, then $\mathscr{X}_{\bar{K}}$ is dominated by a Kummer surface.

Let us now assume that $\rho=20$, let $K$ be the field of fractions of $W(k)$, and let $\bar{K}$ be its algebraic closure. By the classical Shioda-Inose theorem from [SI77] and [I78], there exist isogenous elliptic curves $\widetilde{E}$ and $\widetilde{E}^{\prime}$ with complex multiplication over $\bar{K}$, and a symplectic involution $\imath$ on the Kummer surface $\operatorname{Km}\left(\widetilde{E} \times \widetilde{E}^{\prime}\right)$, such that $\mathscr{X}_{\bar{K}}$ is the desingularization of the quotient $\operatorname{Km}\left(\widetilde{E} \times \widetilde{E}^{\prime}\right) /\langle\imath\rangle$.

STEP 4: This Kummer surface has potential good reduction and $\imath$ extends.

Since elliptic curves with complex multiplication have potential good reduction, there exists a model of $\operatorname{Km}\left(\widetilde{E} \times \widetilde{E}^{\prime}\right)$ over a finite extension $R \supseteq W(k)$ with good reduction that is itself a Kummer surface, say, $\operatorname{Km}\left(\mathscr{E} \times \mathscr{E}^{\prime}\right)($ since $p \neq 2$, we can form the quotient by the sign involution over $R$ without trouble). After possibly enlarging $R$, the involution $\imath$ is defined on the generic fiber $\operatorname{Km}(\mathscr{E} \times \mathscr{E} \prime)_{K}$. Now, $\iota$ extends to an involution on $\operatorname{Km}(\mathscr{E} \times \mathscr{E} \prime)$, see, for example the proof of [LM11, Theorem 2.1]. Since $l$ acts trivially on the global 2 -form of the generic fiber, its extension will act trivially on the global 2 -form of the special fiber, and thus, $r$ extends to a symplectic involution on $\operatorname{Km}\left(\mathscr{E} \times \mathscr{E}^{\prime}\right) \rightarrow \operatorname{Spec} R$. On the geometric generic fiber it has precisely 8 fixed points by [Ni80] or [Mo84, Lemma 5.2], and the same is true for the induced involution on the special fiber by [DK09, Theorem 3.3] (here, we use again that $p \neq 2$ ).

STEP 5: $X$ is the quotient of a Kummer surface by an involution.

After possibly enlarging $R$ again, we may form the quotient $\operatorname{Km}\left(\mathscr{E} \times \mathscr{E}^{\prime}\right) /\langle\imath\rangle$ and resolve the resulting 8 families of $A_{1}$-singularities to obtain a smooth family $\mathscr{Y} \rightarrow \operatorname{Spec} R$. After possibly enlarging $R$ again, the generic fibers of $\mathscr{X}$ and $\mathscr{Y}$ become isomorphic. Since $\mathscr{X}$ and $\mathscr{Y}$ both have good reduction, and their special fibers are not ruled, the special fibers are isomorphic by the Matsusaka-Mumford theorem [MM64, Theorem 2]. This shows the existence of a rational dominant map $\operatorname{Km}\left(E \times E^{\prime}\right) \rightarrow X$, which is generically finite of degree 2 . Here, $E$ and $E^{\prime}$ denote the reductions of $\mathscr{E}$ and $\mathscr{E}^{\prime}$, respectively. The existence of a rational dominant map $X \rightarrow \operatorname{Km}\left(E \times E^{\prime}\right)$, generically finite of degree 2, follows from the corresponding characteristic zero statement as before and we leave the proof to the reader.

STEP 6: Ordinarity and fields of definition.

Since $X$ is ordinary, Frobenius acts bijectively on $H^{2}\left(X, \mathscr{O}_{X}\right)$, from which we conclude that it also acts bijectively on $H^{2}\left(\mathrm{Km}\left(E \times E^{\prime}\right), \mathscr{O}_{\mathrm{Km}\left(E \times E^{\prime}\right)}\right)$ and thus, on $H^{2}\left(E \times E^{\prime}, \mathscr{O}_{E \times E^{\prime}}\right)$. In particular, $E \times E^{\prime}$ is an ordinary Abelian surface, which implies that $E$ and $E^{\prime}$ are ordinary elliptic curves. And finally, since $\widetilde{E}$ and $\widetilde{E}^{\prime}$ are elliptic curves with complex multiplication, they can be defined over $\overline{\mathbb{Q}}$, which implies that $\mathscr{E}, \mathscr{E}^{\prime}, \operatorname{Km}\left(\mathscr{E} \times \mathscr{E}^{\prime}\right)$ and $\imath$ can be defined over $W\left(\overline{\mathbb{F}}_{p}\right)$, which implies that $E, E^{\prime}, \operatorname{Km}\left(E \times E^{\prime}\right)$ and $X$ can be defined over $\overline{\mathbb{F}}_{p}$. This establishes claim (4).

STEP 7: Sketch of the case of Picard rank $\rho=19$. 
As in step 2, let $\mathscr{X} \rightarrow \operatorname{Spec} W(k)$ be a projective lift of $(X, \operatorname{Pic}(X))$. Then, as in step 3 , there exists an Abelian variety $\widetilde{A}$ over some finite extension $L \supseteq K$ and an involution $\imath$ on $\operatorname{Km}(\widetilde{A})$ such that $\operatorname{Km}(\widetilde{A}) / \imath$ and $\mathscr{X}_{K}$ become isomorphic over $\bar{K}$. Since $\mathscr{X}$ has good reduction, the Galois-action of $G_{L}:=\operatorname{Gal}(\bar{K} / L)$ on $H_{\text {ett }}^{2}\left(\mathscr{X}_{\bar{K}}, \mathbb{Q}_{\ell}\right), \ell \neq p$, is unramified. From this, it is not difficult to see that also the $G_{L}$-actions on $H_{\text {èt }}^{2}\left(\operatorname{Km}(\widetilde{A})_{\bar{K}}, \mathbb{Q}_{\ell}\right)$ and $H_{\text {êt }}^{2}\left(\widetilde{A}_{\bar{K}}, \mathbb{Q}_{\ell}\right)$ are unramified. Thus, by the Néron-Ogg-Shafarevich criterion, there exists a smooth model of $\widetilde{A}$ over some finite extension of $W(k)$, whose special fiber $A$ is an Abelian surface. As in step 5 , we find rational dominant maps $\operatorname{Km}(A) \rightarrow X$ and $X \rightarrow \operatorname{Km}(A)$, both of which are generically finite of degree 2 . As in step 6 , we conclude that $A$ is an ordinary Abelian surface. Finally, if $X$ were definable over $\overline{\mathbb{F}}_{p}$, then its geometric Picard rank would be even by [Ar74a, p. 544], a contradiction. This implies that $A$, $\operatorname{Km}(A)$, and $X$ cannot be defined over $\overline{\mathbb{F}}_{p}$ and establishes claim (3).

Remark 2.8. We would like to point out the following analogy between zero and positive characteristic for K3 surfaces with Picard rank 20: over the complex numbers, such surfaces can be defined over $\overline{\mathbb{Q}}$, and thus, form a countable set and have no moduli. In characteristic $p \geq 3$, such surfaces can be defined over $\overline{\mathbb{F}}_{p}$, and again, form a countable set and have no moduli.

\section{CONTINUOUS FAMILIES OF TORSORS}

In this section, we consider Jacobian (quasi-)elliptic fibrations on surfaces in positive characteristic $p$. If the formal Brauer group of the surface is not $p$-divisible, then we construct a deformation of the Jacobian to a non-Jacobian fibration, which is generically a family of torsors under the Jacobian fibration. Using a purely inseparable multisection, we show that the special and the generic fiber of this family are related by a purely inseparable isogeny. Our main result is Theorem 3.6, which is the technical heart of this article. For a K3 surface, such a family exists if only if it is supersingular with Artin invariant $\sigma_{0} \leq 9$, and then, this family can be spread out to a smooth family of supersingular $\mathrm{K} 3$ surfaces over a proper curve such that the generic fiber has Artin invariant $\sigma_{0}+1$.

In order to avoid confusion, let us fix the following terminology.

Definition 3.1. A fibration from a surface onto a curve is said to be of genus 1 if its generic fiber is an integral curve of arithmetic genus 1 . In case the generic fiber is smooth, the fibration is called elliptic, and quasi-elliptic otherwise. Moreover, if the fibration admits a section, it is called Jacobian, and a choice of section, referred to as the zero section, is part of the data.

In characteristic different from 2 and 3, the generic fiber of a genus 1 fibration is automatically smooth by [BM76], and thus, an elliptic fibration.

3.1. Families of torsors arising from formal Brauer groups. For future applications, we extend our setup in this subsection and work with Jacobian genus 1 
fibrations from surfaces that are not necessarily K3. We follow the setup of the articles [AS73] and [Ar74a] by Artin and Swinnerton-Dyer. Let

$$
f: X \rightarrow Y
$$

be a relatively minimal (that is, there are no $(-1)$-curves in the fibers) Jacobian genus 1 fibration, where $X$ is a surface, and $Y$ is a curve, both smooth and proper over an algebraically closed field $k$. Contracting those $(-2)$-curves in the fibers of $f$ that do not intersect the zero section, we obtain the Weierstraß model

$$
f^{\prime}: X^{\prime} \rightarrow Y \text {. }
$$

If $f$ has reducible fibers, then $X^{\prime}$ has rational double point singularities. We denote by $A \subseteq X^{\prime}$ the smooth locus of $X^{\prime}$. As explained in [AS73, Section 1], $A$ has a unique structure $\oplus$ of group scheme over $Y$ : namely, if $P_{1}, P_{2}$ are sections of $A$ over $Y$, then they are Cartier divisors, and $P_{1} \oplus P_{2}$ is the zero locus of a non-zero section of $\mathscr{O}_{X^{\prime}}\left(P_{1}+P_{2}-Z\right)$, where $Z$ denotes the zero section. In case $f$ is an elliptic fibration, we have the following interpretation in terms of Néron models: the smooth locus of $X$ over $Y$ is the Néron model of its generic fiber, and $A$ is its identity component.

Next, let $S$ be the formal spectrum of a local, Noetherian, and complete $k$-algebra with residue field $k$. We want to classify families of torsors under $A$, parametrized by $S$, such that the special fiber is the trivial $A$-torsor. That is, we consider Cartesian diagrams of algebraic spaces

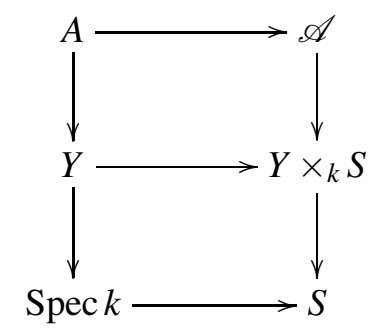

In order to classify such moving torsors, we recall that Artin and Mazur [AM77] studied the functors on local Artinian $k$-algebras with residue field $k$

$$
\begin{array}{clcc}
\Phi_{X / k}^{i}:(\operatorname{Art} / k) & \rightarrow & \text { (Abelian groups) } \\
R & \mapsto \operatorname{ker}\left(H_{\mathrm{et}}^{i}\left(X \times{ }_{k} \operatorname{Spec} R, \mathbb{G}_{m}\right) \rightarrow H_{\mathrm{et}}^{i}\left(X, \mathbb{G}_{m}\right)\right)
\end{array}
$$

see also Section 2.1. We now furthermore assume that $\Phi_{X / k}^{2}$ is pro-representable by a formal group law, which is then called the formal Brauer group and denoted $\widehat{\mathrm{Br}}(X)$, Next, let us recall that there exists a Grothendieck-Leray spectral sequence

$$
E_{2}^{i, j}:=H_{\text {êt }}^{i}\left(Y, R^{j} f_{*}^{\prime} \mathbb{G}_{m}\right) \Longrightarrow H_{\text {ét }}^{i+j}\left(X^{\prime}, \mathbb{G}_{m}\right) .
$$

As Artin explained in [Ar74a, Section 2], the formal structure of $H_{\mathrm{ett}}^{2}\left(X, \mathbb{G}_{m}\right)$ is that of $H_{\text {ét }}^{1}\left(Y, \operatorname{Pic}_{X^{\prime} / Y}\right)$. Using the zero section of $f^{\prime}$, we identify $\operatorname{Pic}_{X^{\prime} / Y}^{0}$ with $A$, and then, it is not difficult to see that moving torsors are closely related to the formal Brauer group. More precisely, we have the following result. 
Proposition 3.2. We keep the notations and assumptions. Let $S:=\operatorname{Spf} R$, where $\left(R, \mathfrak{m}_{R}\right)$ is a local, Noetherian, and complete $k$-algebra with residue field $k$. Let $n \geq 1$ be an integer.

(1) Formal families of A-torsors $\mathscr{A} \rightarrow Y \times_{k} S$, whose special fiber is the trivial $A$-torsor, are classified by the $R$-valued points

$$
\widehat{\operatorname{Br}}(X)(R)
$$

of the formal Brauer group of $X$.

(2) The compactification $A \subseteq X^{\prime}$ extends to a compactification $\mathscr{A} \subseteq \mathscr{X}^{\prime}$, and the formal family $\mathscr{X}^{\prime} \rightarrow Y \times_{k} S$ is algebraizable.

(3) Moreover, $n$-torsion elements of $\widehat{\operatorname{Br}}(X)(R)$ correspond to families as in (1) such that there exists a degree $n$ section of $\operatorname{Pic}_{\mathscr{X}^{\prime} / Y \times{ }_{k} S}$ over $Y \times_{k} S$.

Proof. First, we use the zero section of $f^{\prime}$ to identify $\operatorname{Pic}_{X^{\prime} / Y}^{0}$ with $A$. Then, as explained at the beginning of [Ar74a, Section 2] and in [Ar74a, Proposition (2.1)], the formal structures of $H_{\text {êt }}^{2}\left(X, \mathbb{G}_{m}\right)$ and $H_{\text {êt }}^{2}\left(X^{\prime}, \mathbb{G}_{m}\right)$ are that of $H_{\text {êt }}^{1}\left(Y, \operatorname{Pic}_{X^{\prime} / Y}\right)$. That is, by definition of $\Phi_{X / k}^{2}$ and its pro-representability assumption, we have

$$
\widehat{\operatorname{Br}}(X)(R)=\operatorname{ker}\left(H_{\text {êt }}^{1}\left(Y \times_{k} S, A\right) \stackrel{\text { res }}{\longrightarrow} H_{\text {êt }}^{1}(Y, A)\right),
$$

where res denotes restriction. But then, elements of the right hand side classify $A$-torsors over $Y \times{ }_{k} S$, whose restriction to the special fiber is trivial. This shows claim (1).

Next, we show compactification of $\mathscr{A}$. We set $R_{m}:=R / \mathfrak{m}^{m}$ and $S_{m}:=\operatorname{Spec} R_{m}$. To simplify notations, we denote by $-S_{m}$ the trivial product family $-\times_{\operatorname{Spec} k} S_{m}$. By induction on $m$, we may assume that we have already extended the compactification $A \subseteq X^{\prime}$ to some $\mathscr{A}_{S_{m}} \subseteq \mathscr{X}_{m}^{\prime}$. Blowing up the boundary, we obtain a compactification $\mathscr{A}_{S_{m}} \subseteq \mathscr{Y}_{m}$, whose boundary is a Cartier divisor. As explained in [CLO12, Section 2.1], this latter compactification can be extended to a compactification $\mathscr{A}_{S_{m+1}} \subseteq$ $\mathscr{Y}_{m+1}$. Blowing down $\mathscr{Y}_{m+1}$ to $\mathscr{X}_{m}^{\prime}$ (see, for example, in [CvS09, Theorem 3.1]), we obtain a compactification $\mathscr{A}_{S_{m+1}} \subseteq \mathscr{X}_{m+1}^{\prime}$, which extends $\mathscr{A}_{S_{m}} \subseteq \mathscr{X}_{m}^{\prime}$. Passing to the limit, we obtain the desired compactification $\mathscr{A} \subseteq \mathscr{X}^{\prime}$.

Multiplication by $n$ induces a morphism $A \rightarrow A$ of group schemes over $Y$, and thus, a morphism $\tau_{n}: H_{\mathrm{ett}}^{1}(Y, A) \rightarrow H_{\mathrm{ett}}^{1}(Y, A)$. From the discussion at the end of [AS73, Section 1] it follows that an element in the kernel of $\tau_{n}$ corresponds to an $A$-torsor over $Y$ such that there exists a section of $\operatorname{Pic}_{X^{\prime} / Y}$ over $Y$ of degree $n$. The same holds true with $Y$ replaced by $Y_{S}$, and thus, $n$-torsion elements of $\widehat{\mathrm{Br}}(X)(R)$ correspond to formal families of $A$-torsors over $Y_{S}$ that become trivial over the special fiber, such that there exists a degree- $n$ section of $\operatorname{Pic}_{\mathscr{X}^{\prime} / Y_{S}}$ over $Y_{S}$. This shows claim (3).

It remains to show algebraization. By the established assertion (3), there exists a degree- $n$ section $\overline{\mathscr{L}}$ of $\operatorname{Pic}_{\mathscr{X}^{\prime} / Y_{S}}$ over $Y_{S}$. Since $Y$ is a curve over an algebraically closed field, we have $\operatorname{Br}(Y)=0$ by Tsen's theorem. Since $H^{2}\left(Y, \mathscr{O}_{Y}\right)=0$, we have 
$\widehat{\operatorname{Br}}(Y)=0$, which implies $\operatorname{Br}\left(Y_{S}\right)=0$, and we obtain a short exact sequence

$$
0 \rightarrow \operatorname{Pic}\left(Y_{S}\right) \rightarrow \operatorname{Pic}\left(\mathscr{X}^{\prime}\right) \rightarrow H^{0}\left(Y_{S}, \operatorname{Pic}_{\mathscr{X}^{\prime} / Y_{S}}\right) \rightarrow \underbrace{\operatorname{Br}\left(Y_{S}\right)}_{=0} \rightarrow \ldots
$$

In particular, $\overline{\mathscr{L}}$ lifts to some $\widetilde{\mathscr{L}} \in \operatorname{Pic}\left(\mathscr{X}^{\prime}\right)$. Next, let $E \in \operatorname{Pic}\left(\mathscr{X}^{\prime}\right)$ be the class of a fiber, and then, for every integer $m$, we define $\mathscr{M}_{m}:=\widetilde{\mathscr{L}} \otimes \mathscr{O}_{\mathscr{X}^{\prime}}(m E)$. Since every integral curve on $X^{\prime}$ is either a fiber or a multisection of the fibration, it follows that the restriction $\left.\mathscr{M}_{m}\right|_{X^{\prime}}$ has positive intersection with every integral curve on $X^{\prime}$ if $m \gg 0$. Moreover, for $m \gg 0$, the self-intersection of $\left.\mathscr{M}_{m}\right|_{X^{\prime}}$ is positive. Thus, by the Nakai-Moishezon criterion, for $m \gg 0$, the restriction of $\mathscr{M}_{m}$ to $X^{\prime}$ is an ample invertible sheaf. Therefore, the formal family $\mathscr{X}^{\prime}$ is algebraizable by Grothendieck's existence theorem, which establishes claim (2).

Before proceeding, let us recall a couple of facts about commutative formal group laws, and refer, for example, to [Zi84] for details: if $\widehat{F}$ is a commutative formal group law of dimension $d$ over a field of characteristic zero, then there exists a unique strict isomorphism to $\widehat{\mathbb{G}}_{a}^{d}$, the logarithm of $\widehat{F}$. On the other hand, if $\widehat{F}$ is defined over a field of positive characteristic $p$, then there exists a short exact sequence of commutative formal group laws

$$
0 \rightarrow \widehat{F}^{\mathrm{u}} \rightarrow \widehat{F} \rightarrow \widehat{F}^{\mathrm{bt}} \rightarrow 0,
$$

where $\widehat{F}^{\mathrm{u}}$ is unipotent and $\widehat{F}^{\mathrm{bt}}$ is $p$-divisible [Zi84, Theorem 5.36]. We recall that a formal group law $\widehat{F}$ is called $p$-divisible if multiplication by $p$ is an isogeny, and then, there exists an integer $m \geq 1$ such that the $m$-fold Frobenius

$$
\mathrm{Fr}^{m}: \widehat{F} \rightarrow \widehat{F}^{\left(p^{m}\right)}
$$

factors through multiplication by $p$. The minimal $m$, for which such a factorization exists, is called the height of $\widehat{F}$. On the other extreme, multiplication by $p$ on $\widehat{\mathbb{G}}_{a}$ is zero and thus, this formal group law is of infinite height. More generally, if $\widehat{F}$ is unipotent, then there exists an increasing sequence of formal subgroup laws $0=\widehat{F}_{0} \subset \ldots \subset \widehat{F}_{r}=\widehat{F}$ such that successive quotients are isomorphic to $\widehat{\mathbb{G}}_{a}$, see [Zi84, Theorem 5.37].

This recalled, we have the following statement about formal group laws only, which we need to ensure the existence of non-trivial moving torsor families over Spec $k[[t]]$.

Lemma 3.3. Let $\widehat{F}$ be a formal group law over $k$, and let $\left(R, \mathfrak{m}_{R}\right)$ be a local, Noetherian, and complete $k$-algebra with residue field $k$.

(1) If $p$ does not divide $n$, or $R$ is reduced and $\widehat{F}$ is a p-divisible formal group law, then

$$
\widehat{F}(R)[n]=0 .
$$

(2) If $R$ is reduced and $\mathfrak{m}_{R} \neq 0$, then

$$
\widehat{F}(R)[p] \neq 0 \quad \Leftrightarrow \quad \widehat{F}^{\mathrm{u}} \neq 0,
$$

that is, if and only if $\widehat{F}$ is not p-divisible. 
Proof. If $p \nmid n$, then multiplication by $n$ is injective, and thus, $\widehat{F}(R)[n]=0$. If $\widehat{F}$ is $p$-divisible, say, of finite height $h$, then the $h$-fold Frobenius factors through multiplication by $p$. Since Frobenius is injective on $R$-valued points of $\widehat{F}$ for reduced $R$, this implies $\widehat{F}(R)[p]=0$ and establishes claim (1).

If $\widehat{F}^{\mathrm{u}}=0$ and $R$ is reduced, then $\widehat{F}(R)[p]=0$ by assertion (1). Conversely, if $\widehat{F}$ is not $p$-divisible, then $\widehat{\mathbb{G}}_{a} \subseteq \widehat{F}$. Since $\widehat{\mathbb{G}}_{a}(R)[p]=\mathfrak{m}_{R} \neq 0$, we find $\widehat{F}(R)[p] \neq 0$, which establishes claim (2).

Proposition 3.2 and Lemma 3.3 give us a criterion for the existence of families of $A$-torsors, and ensure compactification and algebraization. The following result gives desingularization, and compares the formal Brauer groups and the Picard groups of special and geometric generic fiber - we note that the latter implies that the family has non-trivial moduli.

Proposition 3.4. We keep the notations and assumptions of Proposition $\$ 3.2$ and assume that $\widehat{\operatorname{Br}}(X)$ is not p-divisible. Set $R:=k[[t]]$ and $S:=\operatorname{Spec} R$, and let

$$
\mathscr{A} \rightarrow Y \times \times_{\text {Spec } k} S \rightarrow S
$$

be a family of A-torsors associated to a non-trivial p-torsion element of $\widehat{\operatorname{Br}}(X)(R)$ as in Proposition 3.2

(1) Let $\mathscr{X}^{\prime} \rightarrow S$ be a compactification as asserted in Proposition 3.2 Then, after possibly replacing $S$ by a finite flat cover, there exists a simultaneous resolution of the singularities

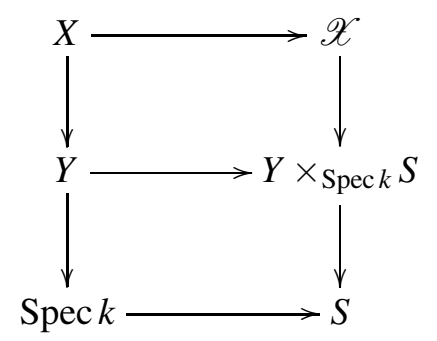

which is smooth and projective over $S$.

(2) Let $\bar{\eta} \in S$ be the geometric generic point. Then, there exists an isomorphism of formal group laws

$$
\widehat{\operatorname{Br}}\left(\mathscr{X}_{\bar{\eta}}\right) \cong \widehat{\operatorname{Br}}(X) \otimes_{k} \kappa(\bar{\eta})
$$

In particular, if $X$ is a supersingular K3 surface, then so is $\mathscr{X}_{\bar{\eta}}$.

(3) Specialization induces a short exact sequence of Picard groups

$$
0 \rightarrow \operatorname{Pic}\left(\mathscr{X}_{\bar{\eta}}\right) \rightarrow \operatorname{Pic}(X) \rightarrow \mathbb{Z} / p \mathbb{Z} \rightarrow 0
$$

whose cokernel is generated by the class of the zero section of $f: X \rightarrow Y$.

Proof. First, $\mathscr{X}^{\prime} \rightarrow S$ is a flat family of surfaces, whose special fiber $X^{\prime}$ has at worst rational double points as singularities. Thus, also the generic fiber has at worst rational double points as singularities by [Li08, Proposition 6.1], and thus, 
after possibly base-changing to a finite flat extension of $S$, there exists a simultaneous resolution of singularities $\mathscr{X} \rightarrow S$ by the main result of [Ar74b]. This establishes claim (1) except for the projectivity statement.

Claim (2) follows from [Ar74a, Proposition (2.1)].

To establish claim (3), we note that there exists a commutative diagram with exact rows, whose vertical arrows are restriction maps:

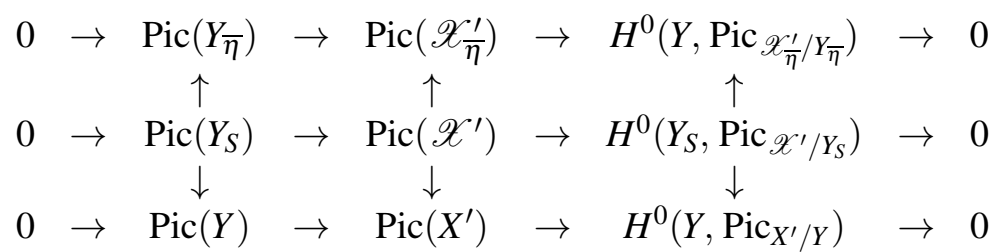

see [AS73, (2.2)], or the proof of Proposition 3.2. Replacing $S$ by a finite flat cover, we may and will assume that the Picard groups of $\mathscr{X}_{\eta}^{\prime}$ and $\mathscr{X}_{\bar{\eta}}^{\prime}$ are isomorphic. Next, it follows from [AS73, Proposition (1.6)] that there exists a commutative diagram of group algebraic spaces over $Y, Y_{S}$ and $Y_{\bar{\eta}}$, respectively:

$$
\begin{array}{rllllllll}
0 & \rightarrow & A_{\bar{\eta}} & \rightarrow & \operatorname{Pic}_{\mathscr{X}_{\bar{\eta}}^{\prime} / Y_{\bar{\eta}}} & \rightarrow & \underline{\mathbb{Z}}_{Y_{\bar{\eta}}} & \rightarrow & 0 \\
& & & & & & \\
0 & \rightarrow & A_{S} & \rightarrow & \operatorname{Pic}_{\mathscr{X}^{\prime} / Y_{S}} & \rightarrow & \underline{\mathbb{Z}}_{Y_{S}} & \rightarrow & 0 \\
& & \downarrow & & \downarrow & \downarrow & & \downarrow \\
0 & \rightarrow & A & \rightarrow & \operatorname{Pic}_{X^{\prime} / Y} & \rightarrow & \underline{\mathbb{Z}}_{Y} & \rightarrow & 0
\end{array}
$$

The class of the zero section $Z$ of $X^{\prime} \rightarrow Y$ in $\operatorname{Pic}\left(X^{\prime}\right)$ defines a splitting of the bottom row. By Proposition 3.2, there exists a degree- $p$ section of Pic $\mathscr{X}^{\prime} / Y_{S}$ over $Y_{S}$. Thus, taking global sections in the previous diagram, we conclude that the image of $H^{0}\left(Y_{S}, \operatorname{Pic}_{\mathscr{X}^{\prime} / Y_{S}}\right)$ inside $H^{0}\left(Y_{S}, \underline{\mathbb{Z}}_{Y_{S}}\right)$ is of index 1 or $p$. However, this index cannot be equal to 1 , since $\mathscr{A} \rightarrow Y_{S}$ is a non-trivial family of $A$-torsors. Combining these observations and the two commutative diagrams, we arrive at a short exact sequence of Abelian groups

$$
0 \rightarrow \operatorname{Pic}\left(\mathscr{X}_{\bar{\eta}}^{\prime}\right) \rightarrow \operatorname{Pic}\left(X^{\prime}\right) \rightarrow \mathbb{Z} / p \mathbb{Z} \rightarrow 0,
$$

where the cokernel is generated by the class of $Z$.

Next, $\operatorname{Pic}(X)$ is generated by the exceptional divisors of the contraction morphism $v: X \rightarrow X^{\prime}$ and $v^{*} \operatorname{Pic}\left(X^{\prime}\right)$, and we have a similar statement for $\operatorname{Pic}\left(\mathscr{X}_{\bar{\eta}}\right)$. Since $\mathscr{A} \rightarrow Y_{S}$ is a family of $A$-torsors, and the special fiber $A$ has no multiple fibers, neither has the generic fiber, and thus, the singular fibers do not change their type by [CD89, Theorem 5.3.1]. In particular, $\mathscr{X}_{\bar{\eta}}^{\prime}$ and $X^{\prime}$ have the same types of rational double points. From this, we deduce that also the cokernel of the specialization homomorphism $\operatorname{Pic}\left(\mathscr{X}_{\bar{\eta}}\right) \rightarrow \operatorname{Pic}(X)$ is cyclic of order $p$, generated by the class of $Z$, which establishes claim (2).

Finally, if $\mathscr{L}$ is an ample invertible sheaf on $X$, then $\mathscr{L}^{\otimes p}$ extends to $\mathscr{X}$, which shows that $\mathscr{X} \rightarrow S$ is projective.

To understand the geometry of moving $A$-torsors better, and to construct purely inseparable multisections, we now inspect the generic fiber more closely. Let $\xi \in Y$ 
be the generic point, and restrict a family $\mathscr{A} \rightarrow Y \times_{k} S$ as in Proposition 3.2 to $\xi \times{ }_{k} S$, that is, we consider

$$
\mathscr{A} \xi:=\mathscr{A} \times{ }_{\left(Y \times_{k} S\right)}\left(\xi \times_{k} S\right) \rightarrow \xi \times_{k} S .
$$

This is a family of torsors under $A_{\xi}:=A \times_{Y} \xi$ over $\xi \times_{k} S$. Let us recall that the relative Frobenius $F: A \rightarrow A^{(p)}$ is a morphism of group schemes over $Y$, whose kernel $A[F]$ is a finite, flat, and infinitesimal group scheme of length $p$ over $Y$.

Proposition 3.5. We keep the notations and assumptions of Proposition 3.2 and assume that $\widehat{\operatorname{Br}}(X)$ is not p-divisible. Set $R:=k[[t]]$ and $S:=\operatorname{Spec} R$, and let

$$
\mathscr{A} \rightarrow Y \times{ }_{k} S \rightarrow S
$$

be a family of A-torsors associated to a non-trivial p-torsion element of $\widehat{\operatorname{Br}}(X)(R)$ as in Proposition 3.2 Then, after possibly replacing $S$ by some finite flat cover

(1) There exists a degree $p$ multisection $D_{\xi} \subset \mathscr{A}_{\xi}$ such that the induced morphism

$$
D_{\xi} \rightarrow \xi \times{ }_{k} S
$$

is finite, flat and radiciel of degree $p$.

(2) More precisely, $D_{\xi} \rightarrow \xi \times_{k} S$ is a family of $A_{\xi}[F]$-torsors. Thus, we obtain an isomorphism

$$
\mathscr{A}_{\xi} \cong\left(A_{\xi} \times{ }_{\left(\xi \times{ }_{k} S\right)} D_{\xi}\right) / A_{\xi}[F],
$$

that is, a description of this family of $A_{\xi}$-torsors as $A_{\xi}[F]$-twist.

PRoOF. If $Z$ denotes the zero section of $f^{\prime}: X^{\prime} \rightarrow Y$, then $\mathscr{O}_{X^{\prime}}(p Z)$ extends to some invertible sheaf $\mathscr{M}$ on $\mathscr{X}^{\prime}$ by Proposition 3.4 . Since $\mathscr{M}$ has degree $p$ on each fiber, Riemann-Roch implies that it has vanishing first cohomology and a $p$-dimensional space of global sections on each fiber. Thus, $\overline{\mathscr{Q}}:=\left(\bar{f}_{*}^{\prime} \mathscr{L}\right)^{\vee}$ is a locally free $\mathscr{O}_{Y \times{ }_{k} S}$-module of rank $p$. This introduced, we recall (for example, from [K105, Section 3]) that relative effective Cartier divisors $D \rightarrow Y_{S}$ such that $\mathscr{O}_{X^{\prime}}(D)$ is isomorphic to $\mathscr{M}$ modulo invertible sheaves coming from $Y_{S}$, correspond to sections of $\mathbb{P}(\overline{\mathscr{Q}}) \rightarrow Y_{S}$. Since the fiber of $\mathbb{P}(\overline{\mathscr{Q}}) \rightarrow Y_{S}$ over $\xi \times_{k} S$ is isomorphic to $\mathbb{P}_{\xi \times_{k} S}^{p-1}$, this already shows the existence of degree- $p$ multisections of $\mathscr{A}_{\xi} \rightarrow \xi \times_{k}$ $S$.

Next, we show that $A[F]$ acts on $\mathbb{P}(\overline{\mathscr{Q}}) \rightarrow Y_{S}$ by translation of relative effective Cartier divisors: the $A$-action on $\mathscr{A}$ induces an $A$-action on the symmetric product $\operatorname{Sym}^{p}(\mathscr{A})$, which we identify with the space of relative effective Cartier divisors of $\mathscr{X}^{\prime} \rightarrow Y_{S}$ of degree $p$ (see, for example, [K105, Remark 9.3.9]). Inside this latter space we have $\mathbb{P}(\overline{\mathscr{Q}}) \rightarrow Y_{S}$, which is stable under the induced $A[F]$-action.

We now determine the schematic fixed point locus of the $A_{\xi}[F]$-action on $\mathbb{P}_{\xi \times S}^{p-1}$. On geometric fibers, a fixed point is of the form $p P$. Thus, depending on the $p$ torsion subgroup scheme of $A_{\xi}$, the fixed point locus is either Artinian of length $p^{2}$ (if $f^{\prime}$ is elliptic), or it is a curve (if $f^{\prime}$ is quasi-elliptic). In any case, the fixed point locus is flat over $\xi \times S$. Thus, after possibly replacing $S$ by a finite flat cover, there exists an $A_{\xi}[F]$-invariant section $D_{\xi}$ of $\mathbb{P}_{\xi \times{ }_{k} S}^{p-1} \rightarrow \xi \times_{k} S$ that specializes to 
$p Z$. By construction, $D_{\xi} \rightarrow \xi \times_{k} S$ is a family of $A_{\xi}[F]$-torsors, and in particular, finite, flat, and radiciel of degree $p$ over $\xi \times{ }_{k} S$, which establishes claim (1). Since the base-change of $\mathscr{A}_{\xi}$ to $D_{\xi}$ trivializes the torsor, we obtain a description of $\mathscr{A}_{\xi}$ as $A_{\xi}[F]$-twist, which establishes claim (2).

We now summarize the results on moving $A$-torsors obtained so far and use a purely inseparable degree- $p$ multisection as established in the previous proposition to show that special and generic fiber of a family of moving $A$-torsors are related by a purely inseparable isogeny. We note that the following theorem is the technical heart of this article.

Theorem 3.6. We keep the notations and assumptions of Proposition 3.2 and assume that $\widehat{\operatorname{Br}}(X)$ is not $p$-divisible. Let $R:=k[[t]]$ and $S:=\operatorname{Spec} R$ and let

$$
\mathscr{A} \rightarrow Y \times{ }_{\operatorname{Spec} k} S \rightarrow S
$$

be a family of A-torsors associated to a non-zero p-torsion element of $\widehat{\operatorname{Br}}(X)(R)$ as in Proposition 3.2 Then, after possibly replacing $S$ by a finite and flat cover,

(1) There exists a compactification and desingularization of $\mathscr{A} \rightarrow Y \times{ }_{k} S$ to a smooth and projective family

$$
\mathscr{X} \rightarrow Y \times_{\operatorname{Spec} k} S \rightarrow S
$$

with special fiber $X$.

(2) Specialization induces a short exact sequence

$$
0 \rightarrow \operatorname{Pic}\left(\mathscr{X}_{\bar{\eta}}\right) \rightarrow \operatorname{Pic}(X) \rightarrow \mathbb{Z} / p \mathbb{Z} \rightarrow 0,
$$

and we have an isomorphism

$$
\widehat{\operatorname{Br}}\left(\mathscr{X}_{\bar{\eta}}\right) \cong \widehat{\operatorname{Br}}(X) \otimes_{k} \kappa(\bar{\eta}),
$$

where $\bar{\eta}$ denotes the geometric generic point of $S$.

(3) There exist a morphism and a rational map

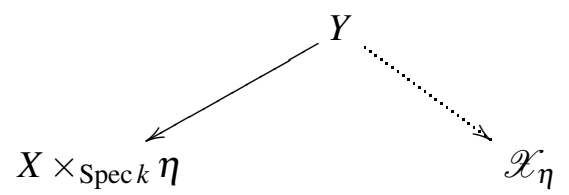

both of which are generically finite and purely inseparable of degree $p$.

(4) There exist rational maps

$$
\mathscr{X}_{\bar{\eta}} \rightarrow X \times_{\text {Spec } k} \bar{\eta} \rightarrow \mathscr{X}_{\bar{\eta}},
$$

both of which are generically finite and purely inseparable of degree $p^{2}$. Thus, $\mathscr{X}_{\bar{\eta}}$ and $X \times_{k} \bar{\eta}$ are purely inseparably isogenous of height 2 .

Proof. We established claims (1) and (2) in Proposition 3.2 and Proposition 3.4.

After possibly replacing $S$ by a finite flat cover, there exists a purely inseparable degree- $p$ multisection $D_{\xi} \subset \mathscr{A}_{\xi}$ by Proposition 3.5, and we denote by $D$ its closure in $\mathscr{X}_{\eta}$. Since $D_{\xi} \rightarrow \xi \times_{k} S$ is finite, flat and radiciel of degree $p$, the same is true 
for $D \rightarrow Y \times_{k} \eta$. Base changing to $D \rightarrow Y \times_{k} \eta$ trivializes the compactified family of $A$-torsors generically, and therefore, we obtain a diagram

$$
X \times_{\operatorname{Spec} k} \eta \longleftarrow\left(X \times_{\operatorname{Spec} k} \eta\right) \times_{\left(Y \times_{k} \eta\right)} D \stackrel{\cong}{\cong} \mathscr{X}_{\eta} \times_{\left(Y \times_{k} \eta\right)} D \longrightarrow \mathscr{X}_{\eta}
$$

where the morphisms on the left and right are purely inseparable of degree $p$, and the rational map in the middle is birational. This establishes claim (3).

Let $F_{\bar{\eta}}: \mathscr{X}_{\bar{\eta}} \rightarrow \mathscr{X}_{\bar{\eta}}^{(p)}$ be the $\bar{\eta}$-linear Frobenius morphism, choose an $\bar{\eta}$-linear isomorphism $\mathscr{X}_{\bar{\eta}}^{(p)} \cong \mathscr{X}_{\bar{\eta}}$, and note that $F_{\bar{\eta}}$ factors through $\mathscr{X}_{\eta} \times_{\left(Y \times{ }_{k} \eta\right)} D$, see also Section 2.2. From this, we obtain a composition

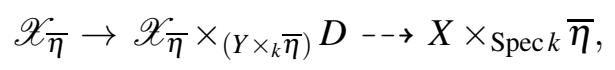

which is a rational map of varieties over $\bar{\eta}$, which is generically finite and purely inseparable of degree $p^{2}$, that is, a purely inseparable isogeny of height 2 . This establishes claim (4).

3.2. Families of supersingular $\mathbf{K} \mathbf{3}$ surfaces. In this subsection we specialize to K3 surfaces. We recall from Section 2.1 that the formal Brauer group $\widehat{\mathrm{Br}}(X)$ of a $\mathrm{K} 3$ surface $X$ is a one-dimensional formal group law. In particular, we have the following equivalences:

$$
\widehat{\operatorname{Br}}(X)^{\mathrm{u}} \neq 0 \Leftrightarrow h(\widehat{\operatorname{Br}}(X))=\infty \Leftrightarrow X \text { is supersingular. }
$$

Thus, by Proposition 3.2 and Lemma 3.3, non-trivial families of moving torsors over Spec $k[[t]]$ associated to a Jacobian (quasi-)elliptic K3 surface can exist only for supersingular K3 surfaces, which renders precise Artin's remark: "The unusual phenomenon of continuous families of homogeneous spaces occurs only for supersingular surfaces" [Ar74a, footnote (2) on p. 552]. The next proposition rephrases Theorem 3.6 in terms of supersingular K3 surfaces.

Proposition 3.7. Let $X \rightarrow \mathbb{P}^{1}$ be a Jacobian (quasi-)elliptic fibration on a supersingular K3 surface over $k$. Then, there exists a smooth and projective family of supersingular elliptic K3 surfaces with non-trivial moduli

$$
\mathscr{X} \rightarrow \mathbb{P}_{S}^{1} \rightarrow S, \quad \text { where } \quad S:=\operatorname{Spec} k[[t]],
$$

whose special fiber is $X \rightarrow \mathbb{P}^{1}$ and that has the following properties:

(1) The Artin invariant of the geometric generic fiber satisfies

$$
\sigma_{0}\left(\mathscr{X}_{\bar{\eta}}\right)=\sigma_{0}(X)+1 .
$$

(2) There exist purely inseparable isogenies of height 2, that is, dominant, rational, and generically finite maps

$$
\mathscr{X}_{\bar{\eta}} \rightarrow X \times_{\operatorname{Spec} k} \bar{\eta} \rightarrow \mathscr{X}_{\bar{\eta}},
$$

whose composition is twice the $\bar{\eta}$-linear Frobenius morphism. 
Proof. By Theorem 3.6, the index of $\operatorname{Pic}\left(\mathscr{X}_{\bar{\eta}}\right)$ in $\operatorname{Pic}(X)$ is equal to $p$, and thus, claim (1) follows from the definition of the Artin invariant. In particular, since the Artin invariants of $X$ and $\mathscr{X}_{\bar{\eta}}$ differ, the family has non-trivial moduli. The remaining assertions are explicitly stated in Theorem 3.6 .

In characteristic $p \geq 5$, supersingular K3 surfaces do not degenerate, that is, have potential good reduction, by a theorem of Rudakov and Shafarevich [RS82]. Thus, the family over Spec $k[[t]]$ described in the previous proposition can be spread out to a smooth family of supersingular K3 surfaces over a smooth and proper curve. More precisely, we have the following result.

Proposition 3.8. If $p \geq 5$ and under the assumptions of Proposition 3.7 there exist a smooth projective curve $C$ over $k$, a closed point $0 \in C$, and a smooth projective family of supersingular $\mathrm{K} 3$ surfaces

$$
\mathscr{Y} \rightarrow C
$$

with the following properties:

(1) After possibly replacing $S$ by a finite flat cover, $\mathscr{X} \rightarrow S$ is the fiber over the completed local ring $\widehat{\mathscr{O}}_{C, 0}$. In particular, $X$ is the fiber over 0 .

(2) Specialization induces an embedding

$$
\operatorname{Pic}(\mathscr{Y} \bar{\eta}) \subset \operatorname{Pic}(X),
$$

which is of index $p$. More precisely, if $E$ denotes a fiber of $X \rightarrow \mathbb{P}^{1}$, and $Z$ the zero-section, then the classes of $E$ and $p Z$ extend to $\operatorname{Pic}(\mathscr{Y} / \bar{\eta})$.

(3) Let $c \in C$ be a point such that the geometric fiber $\mathscr{Y}_{\bar{c}}$ has Artin-invariant $\sigma_{0}(X)+1$. Then, specialization of $E$ to $\mathscr{Y}_{\bar{c}}$ gives rise to a non-Jacobian elliptic fibration. Moreover, there exists a purely inseparable degree-p multisection $D_{\bar{c}}$ on $\mathscr{Y}_{\bar{c}}$, which of class $p Z+k E$ for some $k \geq 2$.

(4) Under the assumptions of (3), there exist purely inseparable isogenies

$$
\mathscr{Y}_{\bar{c}} \rightarrow X \times{ }_{\operatorname{Spec} k} \operatorname{Spec} \kappa(\bar{c}) \rightarrow \mathscr{Y}_{\bar{c}}
$$

both of which are of height 2 .

PROOF. By Artin's approximation theorem [Ar69, Theorem 1.6], the family $\mathscr{X} \rightarrow$ $S$ can be defined over a $k$-algebra of finite type. From there, we spread it out to a projective family $\mathscr{Y} \rightarrow C$, where $C$ is a smooth projective curve over $k$. We denote by $0 \in C$ the point such that the family over the completed ring $\widehat{\mathscr{O}}_{C, 0}$ is $\mathscr{X}$. Since supersingular K3 surfaces in characteristic $p \geq 5$ have potential good reduction by [RS82], we may assume, after possibly replacing $C$ by a finite flat cover, that $\mathscr{Y} \rightarrow C$ is a smooth projective family of supersingular K3 surfaces. This establishes claim (1).

We have a family of elliptic fibrations $\mathscr{X} \rightarrow \mathbb{P}^{1} \times_{k} S \rightarrow S$ (since $p \geq 5$, the fibrations cannot be quasi-elliptic). In particular, the class of $E$ extends from $X$ to $\mathscr{Y}_{\bar{\eta}}$, which, together with Proposition 3.4 establishes claim (2).

Now, let $c \in C$ be a point such that $\sigma_{0}\left(\mathscr{Y}_{\bar{c}}\right)=\sigma_{0}\left(\mathscr{Y}_{\bar{\eta}}\right)$. Then, specialization induces an isomorphism $\operatorname{Pic}(\mathscr{Y} \bar{\eta}) \cong \operatorname{Pic}(\mathscr{Y} \bar{c})$. The elliptic fibration on $\mathscr{Y} \bar{\eta}$ specializes to an elliptic fibration on $\mathscr{Y}_{\bar{c}}$. However, this latter fibration cannot be Jacobian, for 
otherwise there would exist a section, whose class would extend to $\mathscr{Y}_{\bar{\eta}}$, and which would give rise to a section of the original elliptic fibration of $\mathscr{Y} \bar{\eta}$, a contradiction. Since $\mathscr{X} \rightarrow \mathbb{P}^{1} \times{ }_{k} S$ is a family of $A$-torsors, also the Jacobian fibration associated to $\mathscr{Y}_{\bar{c}} \rightarrow \mathbb{P}^{1}$ is $X \rightarrow \mathbb{P}^{1}$.

Next, the degree- $p$ multisection $D \subset \mathscr{Y}_{\bar{\eta}}$ from the proof of Theorem 3.6 specializes to a degree- $p$ multisection $D_{\bar{c}} \subset \mathscr{Y}_{\bar{c}}$. Now, $D_{\bar{c}}$ must be an integral curve, for otherwise, a linear combination of $D_{\bar{c}}$ and $\left(D_{\bar{c}}\right)_{\text {red }}$ would give rise to a relative invertible sheaf on $\mathscr{Y}_{\bar{c}} \rightarrow \mathbb{P}^{1}$ of degree 1 , contradicting the fact that this fibration is not Jacobian. Since the class of $D$ on $\mathscr{Y} \bar{\eta}$ is equal to $p Z$ modulo fiber classes, it must be of class $p Z+k E$ for some integer $k$, and similarly for $D_{\bar{c}}$. Since integral curves on $\mathrm{K} 3$ surfaces have self-intersection number at least -2 , we compute $k \geq 2$. And finally, since $D$ is purely inseparable of degree $p$ over the base, the same is true for its specialization $D_{\bar{c}}$. This establishes claim (3).

Having a non-Jacobian elliptic fibration $\mathscr{Y}_{c} \rightarrow \mathbb{P}^{1}$ with a purely inseparable degree- $p$ multisection $D_{\bar{c}}$, whose associated Jacobian fibration is $X \rightarrow \mathbb{P}^{1}$, the same arguments for the proof of assertion (4) of Theorem 3.6 also show that $\mathscr{Y}_{\bar{c}}$ is related to $X$ by a purely inseparable isogeny of height 2 . This establishes claim (4).

3.3. Jacobian elliptic fibrations on supersingular $\mathbf{K} 3$ surfaces. In order to use Proposition 3.7 we have to show the existence of Jacobian elliptic fibrations on supersingular K3 surfaces. For example, a supersingular K3 surface with Artin invariant $\sigma_{0}=10$ cannot possess such a fibration, for otherwise Proposition 3.7 would produce a supersingular K3 surface with $\sigma_{0}=11$, which is impossible. The next proposition shows that this is the only restriction.

Proposition 3.9. Let $X$ be a supersingular K3 surface with Artin invariant $\sigma_{0}$ in characteristic $p \geq 5$.

(1) If $\sigma_{0} \leq 9$, then $X$ admits a Jacobian elliptic fibration.

(2) If $\sigma_{0}=10$, then $X$ does not admit a Jacobian elliptic fibration.

Remark 3.10. Assertion (2) was already shown by Ekedahl and van der Geer [EG11, Proposition 12.1], as well as by Kondō and Shimada [KS12, Corollary 1.6], but using different methods.

PROOF. We have shown claim (2) in the lines before this proposition.

By [RS78, Section 1], the Artin invariant $\sigma_{0}$ determines $\mathrm{NS}(X)$ up to isometry, and we denote this lattice by $\Lambda_{p, \sigma_{0}}$. Let $U^{\prime}$ be the rank 2 lattice with basis $\{Z, E\}$ and intersection matrix

$$
\left(\begin{array}{cc}
-2 & 1 \\
1 & 0
\end{array}\right)
$$

To show the existence of a Jacobian elliptic fibration on $X$, it suffices to find an isometric embedding of $U^{\prime}$ into $\Lambda_{p, \sigma_{0}}$. Since $U^{\prime}$ is isometric to a hyperbolic plane $U$, and since $\Lambda_{p, \sigma_{0}}$ is a sublattice of $\Lambda_{p, \sigma_{0}-1}$ for every $\sigma_{0} \geq 2$, it suffices to show that $\Lambda_{p, 9}$ contains $U$ in order to establish claim (1). However, this follows from the explicit classification of the lattices $\Lambda_{p, \sigma_{0}}$ in [RS78, Section 1]: namely, there exists an isometry

$$
\Lambda_{p, 9} \cong U \oplus H_{p} \oplus\left(I(-p)^{16}\right)_{*}
$$


where the other lattices are defined and explained in [RS78, Section 1].

Remark 3.11. In characteristic $p \leq 3$, we leave it to the reader to show the following if $X$ is a Shioda-supersingular K3 surface:

(1) If $\sigma_{0} \leq 9$, then $X$ admits a Jacobian genus 1 fibration.

(2) If $\sigma_{0}=10$, then $X$ does not admit a Jacobian genus 1 fibration. Moreover, if $p=3$ and $\sigma_{0}=6$, then $X$ does not admit a Jacobian quasi-elliptic fibration.

3.4. Small Characteristics. Unfortunately, Proposition 3.8 rests on a theorem of Rudakov and Shafarevich [RS82] that supersingular K3 surfaces have potential good reduction, which (currently) requires the assumption $p \geq 5$.

\section{Moduli SPACES}

In this section, we study the moving torsor families from Proposition 3.8 using moduli spaces. In order to avoid technical difficulties, we work with moduli spaces of rigidified K3 crystals rather than moduli spaces of marked supersingular K3 surfaces. As an interesting byproduct, we show that moduli spaces of rigidified $\mathrm{K} 3$ crystals are related to each other by iterated $\mathbb{P}^{1}$-bundles, together with a moduli interpretation. In particular, this gives a new description of these moduli spaces, see Remark 4.4 and Remark 4.7 .

4.1. Recap of Ogus' period map. In this subsection, we shortly review Ogus' articles [Og79] and [Og83]. Let $N$ be a supersingular $K 3$ lattice, that is, the NéronSeveri lattice of a supersingular K3 surface in characteristic $p$. By [RS78, Section 1], such a lattice is determined up to isometry by $p$ and its Artin invariant $\sigma_{0}$.

Definition 4.1. Let $N$ be a supersingular K3 lattice. An $N$-marked supersingular $K 3$ surface is a K3 surface $X$ together with an isometric embedding $N \rightarrow \mathrm{NS}(X)$.

We now assume $p \geq 5$. In [Og83, Theorem (2.7)], Ogus showed the existence of a fine moduli scheme $\mathscr{S}_{N}$ for $N$-marked supersingular K3 surfaces, and proved that it is locally of finite presentation, locally separated, and smooth of dimension $\sigma_{0}(N)-1$ over $\mathbb{F}_{p}$. Moreover, $\mathscr{S}_{N}$ is almost proper, but neither of finite type nor separated over $\mathbb{F}_{p}$. Here, we call a scheme almost proper, if it satisfies the existence part of the valuative criterion for properness with DVR's as test rings.

A $K 3$ crystal of rank 22 consists of a triple $(H,\langle-,-\rangle, \Phi)$, where $H$ is free $W$ module of rank $22,\langle-,-\rangle$ is a symmetric bilinear form on $H$, and $\Phi$ is a Frobeniuslinear endomorphism of $H$, that satisfies the conditions of [Og79, Definition 3.1]. For example, the $F$-crystal arising from $H_{\text {cris }}^{2}$ of a K3 surface, together with the symmetric bilinear form coming from Poincaré duality, is a $\mathrm{K} 3$ crystal. In case $H$ is of slope one, the $\mathrm{K} 3$ crystal is called supersingular. By the crystalline Torelli theorem [Og83, Theorem I], a supersingular K3 surface in characteristic $p \geq 5$ is determined up to isomorphism by its supersingular K3 crystal.

In order to obtain Ogus' period map, we first have to rigidify the $\mathrm{K} 3$ crystals: by definition, the Tate-module of a $\mathrm{K} 3$ crystal $H$ is defined to be $T_{H}:=\{x \in H$ : $\Phi(x)=p x\}$. If $H$ is supersingular, then $T_{H}$ is a free $\mathbb{Z}_{p}$-module of rank 22 , and the bilinear form $\langle-,-\rangle$ on $H$ induces a non-degenerate and non-perfect bilinear 
form on $T_{H}$. Moreover, an $N$-marking of a supersingular $\mathrm{K} 3$ surface induces, via the crystalline Chern map, an isometric embedding of $N$ into the Tate-module of the associated $\mathrm{K} 3$ crystal, which motivates the following definition.

Definition 4.2. Let $N$ be a supersingular K3 lattice. An $N$-rigidified $K 3$ crystal is a pair $\left(\iota: N \rightarrow T_{H}, H\right)$, where $H$ is a $\mathrm{K} 3$ crystal, and $\imath$ is an isometric embedding.

By [Og79, Proposition 4.6], there exists a moduli space $\mathscr{M}_{N}$ of $N$-rigidified K3 crystals, which is smooth and projective of dimension $\sigma_{0}(N)-1$ over $\mathbb{F}_{p}$. We refer to Remark 4.4 and the references given there for details about its geometry. Assigning to an $N$-marked supersingular K3 surface its $N$-rigidified K3 crystal induces a morphism $\pi: \mathscr{S}_{N} \rightarrow \mathscr{M}_{N}$.

In order to get the period map, we have to equip $N$-rigidified K3 crystals with ample cones, and refer to [Og83, Definition 1.15] for definitions. There exists a moduli scheme $\mathscr{P}_{N}$ of $N$-rigidified K3 crystals with ample cones, which is almost proper and locally of finite type over $\mathbb{F}_{p}$. Forgetting the ample cone induces an étale and surjective morphism $f_{N}: \mathscr{P}_{N} \rightarrow \mathscr{M}_{N}$, which is neither of finite type, nor separated. Finally, assigning to an $N$-marked supersingular K3 surface its $N$ rigidified supersingular $\mathrm{K} 3$ crystal together with the ample cone arising from the ample cone of $X$ defines a lift of $\pi$ to a morphism

$$
\tilde{\pi}: \mathscr{S}_{N} \longrightarrow \mathscr{P}_{N} \text {. }
$$

This is Ogus' period map, and it is an isomorphism by [Og83, Theorem III'].

4.2. Moduli spaces of rigidified $\mathbf{K} 3$ crystals. After these preparations, we now interpret Proposition 3.8 in terms of rigidified K3 crystals: if $X$ is a Jacobian elliptic fibration on a supersingular $\mathrm{K} 3$ surface $X$, and $\mathscr{Y} \rightarrow C$ is as in Proposition 3.8, then we obtain orthogonal decompositions

$$
\mathrm{NS}(X) \cong U \oplus \Lambda \quad \text { and } \quad \mathrm{NS}(\mathscr{Y} \bar{\eta}) \cong U(p) \oplus \Lambda .
$$

More precisely, $U$ is the hyperbolic plane generated by the classes of a fiber $E$ and the zero-section $Z$ of the fibration, $\Lambda$ is defined to be $U^{\perp}$ inside $\operatorname{NS}(X)$, and $U(p)$ is the lattice generated by $E$ and $p Z$. Then, we have the following theorem on moduli spaces of rigidified $\mathrm{K} 3$ crystals, which depends on these lattice decompositions only, and which is independent from Section 3 . In Theorem 4.5 below, we will show that it is indeed a manifestation of Proposition 3.8 on the level of K3 crystals.

Theorem 4.3. Let $N$ and $N_{+}$be the supersingular $K 3$ lattices in odd characteristic $p$ of Artin-invariants $\sigma_{0}$ and $\sigma_{0}+1$, respectively. Then, there exists a rank 20 lattice $\Lambda$, and orthogonal decompositions

$$
N \cong U \oplus \Lambda \quad \text { and } \quad N_{+} \cong U(p) \oplus \Lambda,
$$

where $U$ denotes the hyperbolic plane. These decompositions gives rise to a surjective morphism $\varpi_{N}$ of moduli spaces of rigidified K3 crystals with a section $\sigma_{N}$

$$
\begin{gathered}
\mathscr{M}_{N_{+}} \\
\left.\sigma_{N} \uparrow\right|_{\varpi_{N}} ^{\omega_{N}}
\end{gathered}
$$


which turns $\mathscr{M}_{N_{+}}$into a $\mathbb{P}^{1}$-bundle over $\mathscr{M}_{N}$.

PROOF. We proceed in several steps.

STEP 1: Setting up the lattices.

Since $\sigma_{0} \leq 9$, there exists an isometry $N \cong U \oplus \Lambda$ (see, for example, the proof of Proposition 3.9). Next, we choose a basis $\{E, Z\}$ of $U$ such that $E^{2}=0, Z^{2}=-2$, $E \cdot Z=1$. Then, $E$ and $D:=p Z$ span a sublattice of $U$, which is isometric to $U(p)$. Since $U(p) \oplus \Lambda$ is a supersingular K3 lattice of Artin invariant $\sigma_{0}+1$, it is isometric to $N_{+}$by the uniqueness result in [RS78, Section 1]. Thus, we obtain a commutative diagram of embeddings of lattices:

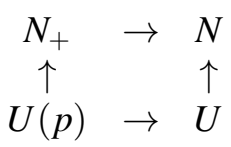

STEP 2: Translation from crystals into characteristic subspaces.

For the explicit computations, it is more convenient to work with characteristic subspaces rather than rigidified K3 crystals, and we refer to [Og79, Proposition 4.3] for the translation between these two points of view. As in loc. cit., we define

$$
N_{0}:=p N^{\vee} / p N \quad \text { and } \quad\left(N_{+}\right)_{0}:=p N_{+}^{\vee} / p N_{+},
$$

which are $\mathbb{F}_{p}$-vector spaces of dimensions $2 \sigma_{0}$ and $2 \sigma_{0}+2$, respectively. Also, the intersection forms turn $p N^{\vee}$ and $p N_{+}^{\vee}$ into sublattices of $N$ and $N_{+}$, respectively. Moreover, by [Og79. Proposition 3.13], the intersection forms on $N$ and $N_{+}$are divisible by $p$ on $p N^{\vee}$ and $p N_{+}^{\vee}$, and induce perfect forms on $N_{0}$ and $\left(N_{+}\right)_{0}$. A straight forward computation shows that the embedding $U(p) \subset N_{+}$induces an isometry $\left(N_{+}\right)_{0} \cong N_{0} \oplus\left(U \otimes \mathbb{F}_{p}\right)$, where $U \otimes \mathbb{F}_{p}$ is generated by the classes of $D$ and $E$. Tensoring the inclusion $N_{+} \subset N$ with $\mathbb{F}_{p}$, we obtain a map $\gamma: N_{+} \otimes \mathbb{F}_{p} \rightarrow$ $N \otimes \mathbb{F}_{p}$, which has a one-dimensional kernel generated by $D$, and whose cokernel is one-dimensional generated by $Z$. Combining the remarks and computations of the previous paragraph, we obtain a commutative diagram of $\mathbb{F}_{p}$-vector spaces

$$
\begin{array}{ccc}
\left(N_{+}\right)_{0} \cong N_{0} \oplus\left(U \otimes \mathbb{F}_{p}\right) & \subset N_{+} \otimes \mathbb{F}_{p} \\
& \downarrow \gamma \\
N_{0} & \subset & N \otimes \mathbb{F}_{p}
\end{array}
$$

For a field $k$ of characteristic $p$, we set $\varphi:=\mathrm{id} \otimes F_{k}^{*}$ on $N_{0} \otimes k$, where $F_{k}$ denotes Frobenius. By [Og79, Definition 3.19], a characteristic subspace of $N_{0} \otimes k$ is a totally isotropic $k$-subvector space $K$, such that $K+\varphi(K)$ is of dimension $\sigma_{0}+1$. It is called strictly characteristic if it is characteristic and moreover $\sum_{i=0}^{\infty} \varphi^{i}(K)$ is equal to $N_{0} \otimes k$. If $A$ is an $\mathbb{F}_{p}$-algebra, then a geneatrix of $N_{0} \otimes A$ is a direct summand of rank $\sigma_{0}$ such that the intersection form restricted to it is identically zero, see [Og79, p. 40]. Finally, a geneatrix is called characteristic if $K+F_{A}^{*}(K)$ is a direct summand of rank $\sigma_{0}+1$. Then, $\mathscr{M}_{N}$ represents the functor taking $A$ to the set of characteristic geneatrices of $N_{0} \otimes A$, see [Og79, Proposition 4.6].

STEP 3: Definition of $\sigma_{N}$. 
By [Og79, Definition 4.1], $\mathscr{M}_{N}$ parametrizes $N$-rigidified K3 crystals, that is, pairs $\left(l: N \rightarrow T_{H}, H\right)$ as in Definition 4.2. Composing $\imath$ with $N_{+} \rightarrow N$ turns an $N$-rigidified K3 crystal into an $N_{+}$-rigidified K3 crystal, which defines a morphism $\sigma_{N}: \mathscr{M}_{N} \rightarrow \mathscr{M}_{N_{+}}$. Translated into geneatrices, this becomes the following: if $A$ is an $\mathbb{F}_{p}$-algebra, and if $K \subset N_{0} \otimes A$ is a characteristic geneatrix, then $\gamma^{-1}(K) \cong$ $K \oplus(D \cdot A)$, and easily seen to be a characteristic geneatrix of $\left(N_{+}\right)_{0} \otimes A$. Using [Og79, Proposition 4.3], it is not difficult to see that the assignment

$$
K \mapsto \gamma^{-1}(K)
$$

describes $\sigma_{N}$ in terms of characteristic geneatrices.

STEP 4: Definition of $\varpi_{N}$.

For an $\mathbb{F}_{p}$-algebra $A$, and a direct summand $K \subseteq\left(N_{+}\right)_{0} \otimes A$, we set

$$
\Gamma_{+}(K):=\operatorname{pr}_{N_{0}}\left(K \cap\left(E^{\perp} \otimes A\right)\right)
$$

where $\mathrm{pr}_{N_{0}}$ denotes the projection $\left(N_{+}\right)_{0} \otimes A \rightarrow N_{0} \otimes A$. A straight forward calculation shows that if $K$ is a characteristic geneatrix of $\left(N_{+}\right)_{0} \otimes A$, then $\Gamma_{+}(K)$ is a characteristic geneatrix of $N_{0} \otimes A$. Thus, the assignment

$$
K \mapsto \Gamma_{+}(K)
$$

defines a morphism $\mathscr{M}_{N_{+}} \rightarrow \mathscr{M}_{N}$ that we denote by $\varpi_{N}$.

STEP 5: $\sigma_{N}$ is a section of $\varpi_{N}$.

If $A$ is an $\mathbb{F}_{p}$-algebra and $K$ is a direct summand of $N_{0} \otimes A$, then it follows from the definitions that $\Gamma_{+}\left(\gamma^{-1}(K)\right)=K$, which shows that $\varpi_{N} \circ \sigma_{N}=$ id.

STEP 6: $\varpi_{N}$ defines a $\mathbb{P}^{1}$-bundle structure.

Using the isomorphism $\left(N_{+}\right)_{0} \cong N_{0} \oplus\left(U \otimes \mathbb{F}_{p}\right)$, we have a projection

$$
\operatorname{pr}_{U}:\left(N_{+}\right)_{0} \rightarrow\left(U \otimes \mathbb{F}_{p}\right) .
$$

Now, let $k$ be an algebraically closed field of characteristic $p$, and let $K_{0} \subset N_{0} \otimes k$ be a characteristic subspace, that is, a $k$-rational point of $\mathscr{M}_{N}$. A straight forward computation shows that if $K_{+} \subset\left(N_{+}\right)_{0} \otimes k$ is characteristic, then $\operatorname{pr}_{U}\left(K_{+} \cap \varphi\left(K_{+}\right)\right)$is one-dimensional. This shows that $K_{+} \cap \varphi\left(K_{+}\right) \cap\left(N_{0} \otimes k\right)$ is $\left(\sigma_{0}-1\right)$-dimensional, where we view $N_{0}$ again as a subspace of $\left(N_{+}\right)_{0}$. In particular, if $\Gamma_{+}\left(K_{+}\right)=K_{0}$, then $K_{+} \cap \varphi\left(K_{+}\right) \cap\left(N_{0} \otimes k\right)=K_{0} \cap \varphi\left(K_{0}\right)$. Thus, every characteristic subspace $K_{+} \subset\left(N_{+}\right)_{0} \otimes k$ with $\Gamma_{+}\left(K_{+}\right)=K_{0}$ contains the $\left(\sigma_{0}-1\right)$-dimensional and totally isotropic subspace $K_{0} \cap \varphi\left(K_{0}\right)$. Let $k_{1}, \ldots k_{\sigma_{0}-1}$ be a basis of $K_{0} \cap \varphi\left(K_{0}\right)$, and choose $v \in K_{0}$ such that $K_{0}=\left\langle v, K_{0} \cap \varphi\left(K_{0}\right)\right\rangle$ and $\varphi\left(K_{0}\right)=\left\langle\varphi(v), K_{0} \cap \varphi\left(K_{0}\right)\right\rangle$. We normalize $v$ such that $\langle v, \varphi(v)\rangle=1$. Then, another straight forward calculation shows that $K_{+} \subset\left(N_{+}\right)_{0} \otimes k$ is characteristic with $\Gamma_{+}\left(K_{+}\right)=K_{0}$ if and only if either $K_{+}=\left\langle K_{0}, E\right\rangle$ or if there exists a unique $\lambda \in k$ such that

$$
K_{+}=\left\langle k_{1}, \ldots, k_{\sigma_{0}-1}, v+\lambda E, v-\lambda \varphi(v)+D+\lambda E\right\rangle .
$$

Thus, the fiber of $\varpi_{N}$ over $K_{0}$ is isomorphic to $\mathbb{P}^{1}$, and since $K_{0}$ was chosen arbitrarily, this shows that all fibers of $\varpi_{N}$ over geometric points of $\mathscr{M}_{N}$ are isomorphic 
to $\mathbb{P}^{1}$. In particular, $\varpi_{N}$ is a conic bundle. Since $\sigma_{N}$ is a section of $\varpi_{N}$, this conic bundle is a $\mathbb{P}^{1}$-bundle.

Remark 4.4. In [Og79, Examples 4.7], Ogus explicitly described $\mathscr{M}_{N}$ in the following cases

\begin{tabular}{cc}
$\sigma_{0}(N)$ & $\mathscr{M}_{N}$ \\
\hline 1 & $\operatorname{Spec} \mathbb{F}_{p^{2}}$ \\
2 & $\mathbb{P}^{1} \times \operatorname{Spec} \mathbb{F}_{p^{2}}$ \\
3 & $\left(\mathbb{P}^{1} \times \mathbb{P}^{1}\right) \times \operatorname{Spec} \mathbb{F}_{p^{2}}$
\end{tabular}

By our previous theorem, $\mathscr{M}_{N}$ is an iterated $\mathbb{P}^{1}$-bundle over $\operatorname{Spec} \mathbb{F}_{p^{2}}$, and we refer to [Og79, Remark 4.8] and [Og79, Theorem 3.21] for further descriptions.

4.3. The moduli interpretation. The previous theorem is about moduli spaces of rigidified $\mathrm{K} 3$ crystals. The following theorem links it to the moving torsor families of supersingular K3 surfaces from Proposition 3.8, and gives a moduli interpretation of $\varpi_{N}$ and $\sigma_{N}$.

Theorem 4.5. We keep the notations and assumptions of Theorem 4.3 Moreover, we assume $p \geq 5$.

(1) Let $X$ be a supersingular K3 surface with $\mathrm{NS}(X) \cong N$, and let $[X] \in \mathscr{M}_{N}$ be the associated $K 3$ crystal. Then, the family

$$
\mathscr{Y} \rightarrow C,
$$

from Proposition 3.8 comes with an $N_{+}$-marking, such that the associated family of $N_{+}$-rigidified $K 3$ crystals maps onto $\varpi_{N}^{-1}([X])$.

(2) Being the fiber over $0 \in C$, the surface $X$ inherits an $N_{+}$-marking, and the corresponding $K 3$ crystal is $\sigma_{N}([X])$.

Proof. We keep the notations from the proof of Theorem 4.3. Given $X$ as in claim (1), we choose the isomorphism $N \cong \mathrm{NS}(X)$ such that $U \subset N$ corresponds to a Jacobian elliptic fibration on $X$, see also the proof of Proposition 3.9. Next, let $\mathscr{Y} \rightarrow C$ be the associated family from Proposition 3.8 . Let $\eta \in C$ be the generic point, set $R:=\mathscr{O}_{C, 0}$, choose a uniformizer $t \in R$, and note that $k(C)$ is the field of fractions of $R$. By Proposition 3.4 the isomorphism $N \cong \mathrm{NS}(X)$ induces an isomorphism $N_{+} \cong \mathrm{NS}\left(\mathscr{Y}_{\eta}\right)$, and, via restriction, the whole family $\mathscr{Y} \rightarrow C$ becomes $N_{+}$-marked. More precisely, we obtain orthogonal decompositions of $N$ and $N_{+}$ and an embedding $N_{+} \subset N$ as in step 1 of the proof of Theorem 4.3 .

Let us now determine the characteristic subspaces associated to $X$ and $\mathscr{Y}_{\eta}$. As explained on [Og83, p. 365], these arise as kernels of the Chern class $c_{\mathrm{dR}}$. We have a commutative diagram

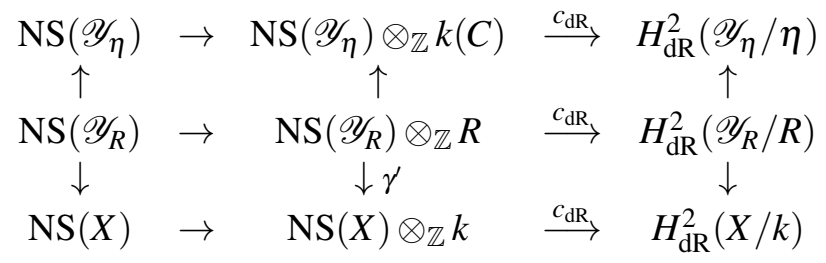


whose vertical arrows are restriction maps. Let $K_{0}^{\prime}:=\varphi^{-1}\left(K_{0}\right) \subset N_{0} \otimes k$ be the characteristic subspace associated to $X$. It is not difficult to see that there exists a lift of $K_{0}^{\prime}$ to an $R$-submodule $\widetilde{K}_{0}^{\prime} \subset N_{+} \otimes R$ of rank $\sigma_{0}$ that is contained in $\operatorname{ker}\left(c_{\mathrm{dR}}\right)$. More precisely, if $k_{1}, \ldots, k_{\sigma_{0}}$ is a basis of $K_{0}^{\prime}$, and $\bar{k}_{i}:=k_{i} \otimes 1 \in N_{0} \otimes R$, there exist lifts of the $k_{i}$ to $\operatorname{ker}\left(c_{\mathrm{dR}}\right)$ of the form

$$
\bar{k}_{i}+t \bar{n}_{i}+\alpha_{i} D+t \beta_{i} E, \quad i=1, \ldots, \sigma_{0},
$$

where $\bar{n}_{i} \in N_{0} \otimes R$, and $\alpha_{i}, \beta_{i} \in R$. There is one more element in $\operatorname{ker}\left(c_{\mathrm{dR}}\right)$, linearly independent from these, and without loss of generality, it is not divisible by $t$ and lies in the kernel of $\gamma^{\prime}$. Thus, we may choose it to be of the form

$$
t \bar{n}_{0}+D+t \beta E,
$$

where $\bar{n}_{0} \in N_{0} \otimes R$ and $\beta \in R$. Since these $\sigma_{0}+1$ elements lie inside $\operatorname{ker}\left(c_{d R}\right)$, they form a totally isotropic subspace. After some tedious computations exploiting this isotropy, we find that $\operatorname{ker}\left(c_{d R}\right)$ contains a free $R$-submodule $\widetilde{K}_{+}^{\prime}$ of rank $\sigma_{0}+1$ generated by elements of the form

$$
\begin{array}{cc}
\bar{k}_{i} & +t \mu_{i} E \\
t \bar{n}_{0} & +D+t \beta E
\end{array}
$$

Simply for dimensional reasons, $\widetilde{K}_{+}^{\prime} \otimes \overline{k(C)} \subset\left(N_{+}\right)_{0} \otimes \overline{k(C)}$ is the characteristic subspace associated to $\mathscr{Y} \bar{\eta}$.

Using this explicit description, we compute $\gamma^{\prime}\left(\widetilde{K}_{+}^{\prime}\right)=K_{0}^{\prime}$ and $\Gamma_{+}\left(\widetilde{K}_{+}^{\prime}\right)=K_{0}^{\prime} \otimes_{k} R$, where $\Gamma_{+}$is defined as in the proof of step 4 of Theorem 4.3, In particular, the classifying map $f_{C}: C \rightarrow \mathscr{M}_{N_{+}}$maps to the fiber $\varpi_{N}^{-1}([X])$. Since the fibers of $\varpi$ and $C$ are proper irreducible curves and $f_{C}$ is not constant, $f_{C}$ maps surjectively onto $\varpi_{N}^{-1}([X])$, which establishes claim (1). The fiber over $0 \in C$ is isomorphic to $X$, and the $N_{+}$-marking of $\mathrm{NS}(X)$ induced from the $N_{+}$-marking of the family $\mathscr{Y} \rightarrow C$ arises via $N_{+} \rightarrow N \cong \mathrm{NS}(X)$. Thus, by the definition of $\sigma_{N}$ in step 3 of the proof of Theorem 4.3, the associated $N_{+}$-rigidified K3 crystal is $\sigma_{N}([X])$, which establishes claim (2).

Let us give an immediate corollary of Theorem 4.3 and Theorem 4.5 , which is actually everything we will need to prove the results of the next section.

Corollary 4.6. Let $Y$ be a supersingular $K 3$ surface in characteristic $p \geq 5$ with $\sigma_{0}(Y) \geq 2$. Then, there exists a supersingular $K 3$ surface $X$ with $\sigma_{0}(X)=\sigma_{0}(Y)-1$ that is purely inseparably isogenous of height 2 to $Y$.

Proof. By Theorem 4.3 and Theorem 4.5, there exists a family $\mathscr{Y} \rightarrow C$ of $N_{+^{-}}$ marked supersingular $\mathrm{K} 3$ surfaces, where $\sigma_{0}\left(N_{+}\right)=\sigma_{0}(Y)$, such that $Y$ is a member of this family. Let $X$ be the special fiber over $0 \in C$, and then, the assertion follows from Proposition 3.8

Remark 4.7. The unirationality of $\mathscr{M}_{N}$ is clear from Ogus' description [Og79, Theorem 3.21], whereas our description as iterated $\mathbb{P}^{1}$-bundle is new. It is likely that Theorem 4.3 and Theorem 4.5 extend in some form to the moduli spaces $\mathscr{S}_{N}$ 
of $N$-marked supersingular K3 surfaces. However, since these latter spaces are neither of finite type nor separated, the proofs and maybe even the statements would probably be rather technical and involved. Much better behaved are moduli spaces of polarized K3 surfaces. In [Li13, Section 9], Lieblich announced the existence of families of supersingular K3 surfaces over $\mathbb{A}^{1}$ using moduli spaces of twisted sheaves. As an application, he announces the uniruledness of the supersingular loci of moduli spaces of polarized K3 surfaces. For complex K3 surfaces, nontrivial families over $\mathbb{P}^{1}$, whose general member is not algebraic, arise from twistor spaces, see [Hu03, Section 25]. For example, Markman [Mar13, Section 7] and Verbitsky [Ve13, Section 1.4] studied twistor spaces together with Lagrangian fibrations, which is similar to our moving torsor families.

4.4. Small Characteristics. The results of this section build on Ogus' articles [Og79] and [Og83]. In [Og79], he develops the theory of supersingular K3 crystals, and the assumption $p \geq 3$ is built in from the very beginning: quadratic and symplectic forms play an important role, which is why characteristic 2 is excluded. In [Og83], $p \geq 5$ had to be assumed, not only because it rests on [Og79], but also since it needs the theorem of Rudakov-Shafarevich [RS82] on potential good reduction of supersingular K3 surfaces, see [Og83, p. 364].

\section{SUPERSingular K3 SURFACES ARE UNIRATIONAL}

In this section, we prove that supersingular K3 surfaces in characteristic $p \geq 5$ are related by purely inseparable isogenies, which is an analog of the Shioda-Inose structure theorem for singular K3 surfaces, see Theorem 2.6. Since Shioda [Sh77b] showed that supersingular Kummer surfaces are unirational, we deduce the ArtinRudakov-Shafarevich-Shioda conjecture on unirationality of all supersingular K3 surfaces. Finally, we treat unirationality of Enriques surfaces.

5.1. Isogenies between supersingular $\mathbf{K} 3$ surfaces. We now come to the main theorem of this article, which is a structure result for supersingular K3 surfaces. We note that Rudakov and Shafarevich conjectured this already in Question 8 at the end of [RS78]. We refer to Section 2.2 for the connection with a conjecture of Shafarevich about isogenies between complex K3 surfaces.

Theorem 5.1. Let $X$ and $X^{\prime}$ be supersingular K3 surfaces with Artin invariants $\sigma_{0}$ and $\sigma_{0}^{\prime}$ in characteristic $p \geq 5$.

(1) There exist purely inseparable isogenies

$$
X \rightarrow X^{\prime} \rightarrow X \text {, }
$$

both of which are of height $2 \sigma_{0}+2 \sigma_{0}^{\prime}-4$.

(2) Let $E$ be a supersingular elliptic curve. Then, there exist isogenies

$$
\mathrm{Km}(E \times E) \rightarrow X \rightarrow \operatorname{Km}(E \times E),
$$

both of which are purely inseparable of height $2 \sigma_{0}-2$. 
PROOF. If $\sigma_{0} \geq 2$, then there exists a supersingular K3 surface with Artin invariant $\sigma_{0}-1$ that is purely inseparable isogenous of height 2 to $X$ by Corollary 4.6 . By induction, we obtain a purely inseparable isogeny $\varphi$ of height $2 \sigma_{0}-2$ from $X$ to a supersingular K3 surface with Artin invariant $\sigma_{0}=1$. However, there exists only one such surface, namely the Kummer surface $\operatorname{Km}(E \times E)$, where $E$ is a supersingular elliptic curve [Og79, Corollary (7.14)]. Since the $\left(2 \sigma_{0}-2\right)$-fold Frobenius of $X$ factors through $\varphi$, we obtain claim (2).

By the established claim (2), there exists a purely inseparable isogeny $\varphi^{\prime}$ : $\operatorname{Km}(E \times E) \rightarrow X^{\prime}$ of height $2 \sigma_{0}^{\prime}-2$. Then, $\varphi^{\prime} \circ \varphi$ is a purely inseparable isogeny $X \rightarrow X^{\prime}$ of height $2 \sigma_{0}+2 \sigma_{0}^{\prime}-4$. As before, the $\left(2 \sigma_{0}+2 \sigma_{0}^{\prime}-4\right)$-fold Frobenius of $X$ factors through $\varphi^{\prime} \circ \varphi$ and we obtain claim (1).

Remark 5.2. Naively, one might expect that $\mathrm{K} 3$ surfaces of Picard rank $\geq \rho$ form a codimension $\rho$ subset inside the moduli space. This expectation is fulfilled for singular K3 surfaces $(\rho=20)$, since they form a countable set. But then, one would expect that K3 surfaces with $\rho=22$ should not exist at all, and the fact that they come in 9-dimensional families is even more puzzling. However, by Theorem 5.1 , there exists only one supersingular K3 surface in every positive characteristic up to purely inseparable isogeny. By Proposition 3.8 , these isogenies come in families, which gives an explanation why supersingular K3 surfaces form 9-dimensional moduli spaces.

5.2. Supersingular K3 surfaces are unirational. Since Shioda [Sh77b] showed that supersingular Kummer surfaces are unirational, the previous theorem implies the conjecture of Artin, Rudakov, Shafarevich, and Shioda.

Theorem 5.3. Supersingular K3 surfaces in characteristic $p \geq 5$ are unirational.

PROOF. In odd characteristic, supersingular Kummer surfaces are unirational by [Sh77b, Theorem 1.1]. The assertion then follows from Theorem 5.1.

We recall that a surface is called a Zariski surface if there exists a dominant, rational, and purely inseparable map of degree $p$ from $\mathbb{P}^{2}$ onto it. Although the map from $\mathbb{P}^{2}$ onto a supersingular Kummer surface constructed by Shioda in [Sh77b] is inseparable, it is not purely inseparable. Using a different construction, Katsura [Ka87, Theorem 5.10] showed that supersingular Kummer surfaces with $\sigma_{0}=1$ in characteristic $p \not \equiv 1 \bmod 12$ are Zariski surfaces. This strengthens Theorem 5.3, and gives a partial answer to a question of Rudakov and Shafarevich, who asked and actually doubted whether supersingular K3 surfaces are purely inseparably unirational, see Question 6 at the end of [RS78].

Corollary 5.4. A supersingular $K 3$ surface in characteristic $p \geq 5$ with $p \not \equiv 1$ mod 12 is purely inseparably unirational.

In Section 2.1, we discussed different notions of supersingularity for K3 surfaces and the relation to the Tate-conjecture. Combining Theorem 2.3 and Theorem 5.3, we obtain the following equivalence.

Theorem 5.5. For a $K 3$ surface $X$ in characteristic $p \geq 5$, the following conditions are equivalent: 
(1) $X$ is unirational.

(2) The Picard rank of $X$ is 22.

(3) The formal Brauer group of $X$ is of infinite height.

(4) For all $i$, the $F$-crystal $H_{\text {cris }}^{i}(X / W)$ is of slope $i / 2$.

Proof. If $X$ is unirational, then its Picard rank is 22 by [Sh74, Corollary 2], which establishes $(1) \Rightarrow(2)$. The converse direction $(2) \Rightarrow(1)$ is Theorem 5.3 , The equivalences $(2) \Leftrightarrow(3) \Leftrightarrow(4)$ are Theorem 2.3 .

5.3. Enriques surfaces. As a consequence of Theorem 5.3, we now characterize the unirational ones among Enriques surfaces, which generalizes a result of Shioda [Sh77b, Theorem 3.3].

Theorem 5.6. An Enriques surface $X$ in characteristic $p \geq 2$ is unirational if and only if

(1) $p=2$ and $X$ is not singular (that is, $\mathrm{Pic}_{X / k}^{\tau} \neq \mu_{2}$ ), or

(2) $p \neq 2$ and the covering $K 3$ surface is supersingular.

Proof. Assertion (1) is shown in [CD89, Corollary I.1.3.1].

By [Sh77b, Lemma 3.1], an Enriques surface $X$ in characteristic $p \geq 3$ is unirational if and only if its covering K3 surface $\widetilde{X}$ is unirational. Thus, if $p \geq 5$, then assertion (2) follows from Theorem 5.5. If $p=3$ and $X$ is unirational, then $\widetilde{X}$ is unirational, and thus, supersingular. Conversely, if $p=3$ and $\widetilde{X}$ is supersingular, then $\sigma_{0}(\widetilde{X}) \leq 5$ by [Ja13, Corollary 3.4] and thus, $\widetilde{X}$ is unirational by [RS78], which implies the unirationality of $X$.

5.4. Small Characteristics. As in Section 3.4 and Section 4.4 let us discuss what we know and do not know in characteristic $p \leq 3$.

(1) Using quasi-elliptic fibrations, Rudakov and Shafarevich [RS78] showed that Shioda-supersingular K3 surfaces in characteristic 2 and supersingular K3 surfaces with $\sigma_{0} \leq 6$ in characteristic 3 are Zariski surfaces, and thus, unirational. Therefore, the question remains whether supersingular K3 surfaces with $\sigma_{0} \geq 7$ in characteristic 3 are unirational. By Proposition 3.7 together with the comments made in Section 3.4, there exists at least a 6-dimensional family of unirational $\mathrm{K} 3$ surfaces with $\sigma_{0}=7$ in characteristic 3.

(2) Theorem 5.1 rests on Corollary 4.6, and we refer to Section 4.4 for details. On the other hand, quasi-elliptic K3 surfaces are Zariski surfaces, and thus, related by purely inseparable isogenies.

(3) The implication $(1) \Rightarrow(2)$ of Theorem 5.5 holds in any characteristic and we discussed it converse above. The implication $(2) \Rightarrow(3)$ holds in any characteristic, and its converse would follow from the Tate-conjecture for $\mathrm{K} 3$ surfaces, which is true in characteristic 3 by [MP13]. The equivalence $(3) \Leftrightarrow(4)$ holds in every characteristic. 
In particular (see also Section 4.4), once supersingular K3 surfaces in characteristic 3 are shown to have potential good reduction, the results of this section will also hold in characteristic 3 .

\section{APPENDIX A. ERRATUM}

As was pointed out by Bragg and Lieblich in [BL22], the proof of Proposition 3.5 contains a mistake, which has the effect that the main result, the unirationality of supersingular K3 surfaces Theorem 5.3, remains a conjecture.

Degree p multisections. More precisely, the proof only shows the following:

Proposition A.1. We keep the notations and assumptions of Proposition 3.2 and assume that $\widehat{\operatorname{Br}}(X)$ is not $p$-divisible. Set $R:=k[[t]]$ and $S:=\operatorname{Spec} R$, and let

$$
\bar{A} \rightarrow Y \times{ }_{k} S \rightarrow S
$$

be a family of A-torsors associated to a non-trivial p-torsion element of $\widehat{\mathrm{Br}}(X)(R)$ as in Proposition 3.2. Then, after possibly replacing $S$ by some finite flat cover

(1) There exists a degree $p$ multisection $D_{\xi} \subset \bar{A}_{\xi}$ such that the induced morphism

$$
D_{\xi} \rightarrow \xi \times{ }_{k} S
$$

is finite and flat of degree $p$.

(2) More precisely, $D_{\xi} \rightarrow \xi \times_{k} S$ arises from a family of $A_{\xi}[p]$-torsors.

In particular, contrary to what was claimed in Proposition 3.5, one may not be able to choose the degree $p$ multisection $D_{\xi}$ to be radicial, that is, purely inseparable, over $\xi \times{ }_{k} S$.

The strategy of establishing Theorem 5.3 consisted in showing that any two supersingular K3 surfaces in characteristic $p \geq 5$ can be connected by one-dimensional families of the type as constructed in Section 3 and then, that unirationality is preserved in these families. This reduced the unirationality conjecture to the showing that there exists at least one unirational K3 surface in each characteristic, where one can use Kummer surfaces, whose unirationality was established by Shioda.

Now, in order to show that unirationality is preserved in these one-dimensional families, one needs Assertion (4) of Theorem 3.6, whose proof requires that one can find degree $p$ multisections in Proposition 3.5 that are radicial over the base. Without this, one can only conclude that any two supersingular K3 surfaces in characteristic $p \geq 5$ are connected by a sequence of very special degree $p$ correspondences (as in Assertion (3) of Theorem 3.6), which is not sufficient for unirationality.

Fibration structure. Moreover, the proof of Theorem 4.3 contains a mistake. In fact, the proof only shows the following:

Theorem A.2. Let $N$ and $N_{+}$be the supersingular K3 lattices in odd characteristic p of Artin-invariants $\sigma_{0}$ and $\sigma_{0}+1$, respectively. Then, there exists a rank 20 lattice $\Lambda$, and isometries

$$
N \cong U \oplus \Lambda \quad \text { and } \quad N_{+} \cong U(p) \oplus \Lambda,
$$


where $U$ denotes the hyperbolic plane. This decomposition gives rise to a dominant rational map $\varpi_{N}$ of moduli spaces of rigidified $K 3$ crystals with a section $\sigma_{N}$

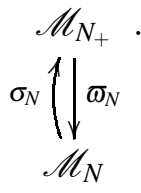

More precisely,

(1) this map is defined on the open set of $\mathscr{M}_{N+1}$ of rigidified $K 3$ crystals with Artin invariant $\sigma_{0}+1$ and maps surjectively onto the open set of rigidified $K 3$ crystals of Artin invariant $\sigma_{0}$.

(2) The normalization of the geometric generic fibre is $\mathbb{P}^{1}$. (In general, $\varpi_{N}$ may not be generically smooth.)

In the proof, the map is only defined on geometric points and extension to a morphism would require more arguments. In particular, the computations in Step 6 of the proof only show that the normalisation of the geometric generic fibre is isomorphic to $\mathbb{P}^{1}$.

In particular, the table Remark 4.4 has to be corrected as follows: for $\sigma_{0}(N)=3$, the moduli space $\mathscr{M}_{N}$ consists geometrically of two copies of the Fermat hypersurface of degree $(p+1)$ in $\mathbb{P}^{3}$. In this case, $\bar{\varpi}_{N}$ arises geometrically by choosing a pencil of planes through a line of this surface and then, one obtains a fibration over $\mathbb{P}^{1}$, whose geometric generic fibre is a singular rational curve that is not smooth. This gives an example, where the geometric generic fibre of $\varpi_{N}$ is not smooth, and in particular, not isomorphic to $\mathbb{P}^{1}$.

\section{REFERENCES}

[Ar69] M. Artin, Algebraization of formal moduli: I, Global analysis (papers in honor of K. Kodaira), Univ. of Tokyo Press, 21-71 (1969).

[Ar74a] M. Artin, Supersingular K3 surfaces, Ann. Sci. École Norm. Sup. (4) 7, 543-567 (1974).

[Ar74b] M. Artin, Algebraic construction of Brieskorn's resolutions, J. Algebra 29, 330-348 (1974).

[AM77] M. Artin, B. Mazur, Formal groups arising from algebraic varieties, Ann. Sci. École Norm. Sup. 10, 87-131 (1977).

[AS73] M. Artin, H. P. F. Swinnerton-Dyer, The Shafarevich-Tate conjecture for pencils of elliptic curves on K3 surfaces, Invent. Math. 20, 249-266 (1973).

[BM76] E. Bombieri, D. Mumford, Enriques' Classification of Surfaces in Char. p, III, Invent. Math. 35, 197-232 (1976).

[BL22] D. Bragg, M. Lieblich, Perfect points on curves of genus 1 and consequences for supersingular K3 surfaces, Compos. Math. 158 (2022), no. 5, 1052-1083.

[Ch13] F. Charles, The Tate conjecture for K3 surfaces over finite fields, Invent. Math. 194, 119-145 (2013).

[Ch10] X. Chen, Self rational maps of K3 surfaces, arXiv:1008.1619](2010).

[CD89] F. R. Cossec, I. V. Dolgachev, Enriques Surfaces I, Progress in Mathematics 76, Birkhäuser, 1989.

[CLO12] B. Conrad, M. Lieblich, M. Olsson, Martin, Nagata compactification for algebraic spaces, J. Inst. Math. Jussieu 11, 747-814 (2012).

[CvS09] S. Cynk, D. van Straten, Small resolutions and non-liftable Calabi-Yau threefolds, manuscripta math. 130, 233-249 (2009). 
[De81] P. Deligne, Relèvement des surfaces K3 en caractéristique nulle, Lecture Notes in Math. 868, Algebraic surfaces (Orsay, 1976-78), 58-79, Springer 1981.

[DK09] I. V. Dolgachev, J. Keum, Finite groups of symplectic automorphisms of K3 surfaces in positive characteristic, Ann. of Math. (2) 169, 269-313 (2009).

[Ek87] T. Ekedahl, Foliations and inseparable morphisms, Algebraic geometry, Bowdoin 1985, 139-149, Proc. Sympos. Pure Math. 46 (1987).

[EG11] T. Ekedahl, G. van der Geer, Cycle Classes on the Moduli of K3 surfaces in positive characteristic, arXiv:1104.3024(2011).

[Hu03] D. Huybrechts, Compact hyperkähler manifolds, Calabi-Yau manifolds and related geometries (Nordfjordeid, 2001), 161-225, Springer (2003).

[Ig60] J. I. Igusa, Betti and Picard numbers of abstract algebraic surfaces, Proc. Nat. Acad. Sci. USA 46, 724-726 (1960).

[I179] L. Illusie, Complexe de de Rham-Witt et cohomologie cristalline, Ann. Sci. École Norm. Sup. 12, 501-661 (1979).

[I105] L. Illusie, Grothendieck's existence theorem in formal geometry, Math. Surveys Monogr. 123, Fundamental algebraic geometry, 179-233, AMS 2005.

[178] H. Inose, Defining equations of singular K3 surfaces and a notion of isogeny, Proceedings of the International Symposium on Algebraic Geometry (Kyoto Univ., Kyoto, 1977), 495502, Kinokuniya Book Store, 1978.

[I-L13] H. Ito, C. Liedtke, Elliptic K3 surfaces with $p^{n}$-torsion sections, J. Algebraic Geom. 22, 105-139 (2013).

[Ja13] J. Jang, Néron-Severi group preserving lifting of $K 3$ surfaces and applications, arXiv:1306.1596 (2013).

[Ka87] T. Katsura, Generalized Kummer surfaces and their unirationality in characteristic p, J. Fac. Sci. Univ. Tokyo Sect. IA Math. 34, 1-41 (1987).

[K166] S. L. Kleiman, Toward a numerical theory of ampleness, Ann. of Math. (2) 84, 293-344 (1966).

[K105] S. L. Kleiman, The Picard scheme, Fundamental algebraic geometry, 235-321, Math. Surveys Monogr. 123, AMS 2005.

[KS12] S. Kondo, I. Shimada, On certain duality of Néron-Severi lattices of supersingular K3 surfaces and its application to generic supersingular K3 surfaces, arXiv:1212.0269 (2012).

[LM11] M. Lieblich, D. Maulik, A note on the cone conjecture for K3 surfaces in positive characteristic, arXiv:1102.3377 (2011).

[Li13] M. Lieblich, On the ubiquity of twisted sheaves, Birational geometry, rational curves, and arithmetic, 205-227, Springer (2013).

[Li14] M. Lieblich, On the unirationality of supersingular K3 surfaces, arXiv:1403.3073 (2014).

[Li08] C. Liedtke, Algebraic Surfaces of General Type with Small $c_{1}^{2}$ in Positive Characteristic, Nagoya Math. J. 191, 111-134 (2008).

[Ma13] S. Ma, On K3 surfaces which dominate Kummer surfaces, Proc. Amer. Math. Soc. 141, 131-137 (2013).

[MP13] K. Madapusi Pera, The Tate conjecture for K3 surfaces in odd characteristic, arXiv:1301.6326 (2013).

[Mar13] E. Markman, Lagrangian fibrations of holomorphic-symplectic varieties of $\mathrm{K} 3^{[n]}$-type, arXiv:1301.6584 (2013).

[MM64] T. Matsusaka, D. Mumford, Two fundamental theorems on deformations of polarized varieties, Amer. J. Math. 86, 668-684 (1964).

[Mau12] D. Maulik, Supersingular K3 surfaces for large primes, arXiv:1203.2889 (2012).

[Mo84] D. R. Morrison, On K3 surfaces with large Picard number, Invent. Math. 75, 105-121 (1984).

[Mo87] D. R. Morrison, Isogenies between Algebraic Surfaces with Geometric Genus One, Tokyo J. of Math. 10, 179-187 (1987).

[Mu87] S. Mukai, On the moduli space of bundles on K3 surfaces. I, Vector bundles on algebraic varieties (Bombay, 1984), 341-413, Tata Inst. Fund. Res. Stud. Math., 11, 1987. 
[Ni80] V. V. Nikulin, Finite automorphism groups of Kähler K3 surfaces, Trans. Moscow Math. Soc. 38, 75-135 (1980).

[Ni87] V. V. Nikulin, On correspondences between surfaces of K3 type, Izv. Akad. Nauk SSSR Ser. Mat. 51 (1987), translation in Math. USSR-Izv. 30, 375-383 (1988).

[Ni91] V. V. Nikulin, On rational maps between K3 surfaces, Constantin Carathéodory: an international tribute, Vol. I, II, 964-995, World Sci. Publ., 1991.

[Ny] N. O. Nygaard, The Tate conjecture for ordinary K3 surfaces over finite fields, Invent. Math. 74, 213-237 (1983).

[Og79] A. Ogus, Supersingular K3 crystals, Journées de Géométrie Algébrique de Rennes, Vol. II, Astérisque 64, 3-86 (1979).

[Og83] A. Ogus, A crystalline Torelli theorem for supersingular K3 surfaces, Arithmetic and geometry, Vol. II, 361-394, Progress in Mathematics 36, Birkhäuser, 1983.

[PS06] D. T. Pho, I. Shimada, Unirationality of certain supersingular K3 surfaces in characteristic 5, Manuscripta Math. 121, 425-435 (2006).

[RS78] A. N. Rudakov, I. R. Shafarevich, Supersingular K3 surfaces over fields of characteristic 2, Izv. Akad. Nauk SSSR 42, 848-869 (1978), Math. USSR, Izv. 13, 147-165 (1979).

[RS81] A. N. Rudakov, I. R. Shafarevich, Surfaces of type K3 over fields of finite characteristic, Current problems in mathematics 18, 115-207, Akad. Nauk SSSR, Vsesoyuz. Inst. Nauchn. i Tekhn. Informatsii, Moscow, 1981.

[RS82] A. N. Rudakov, I. R. Shafarevich, On the degeneration of K3 surfaces over fields of finite characteristic, Math. USSR Izv. 18, 561-574 (1982).

[Sh71] I. R. Shafarevich, Le théorème de Torelli pour les surfaces algébriques de type K3, ICM Nice 1970, Vol. 1, 413-417, Gauthier-Villars 1971.

[Sh73] T. Shioda, Algebraic cycles on certain K3 surfaces in characteristic p, Manifolds-Tokyo 1973 (Proc. Internat. Conf., Tokyo, 1973), 357-364, Univ. Tokyo Press 1975.

[Sh74] T. Shioda, An example of unirational surfaces in characteristic p, Math. Ann. 211, 233-236 (1974).

[Sh77b] T. Shioda, Some results on unirationality of algebraic surfaces, Math. Ann. 230, 153-168 (1977).

[Sh79] T. Shioda, Supersingular K3 surfaces, Algebraic geometry, Springer Lecture Notes 732, 564-591 (1979).

[SI77] T. Shioda, H. Inose, On singular K3 surfaces, Complex analysis and algebraic geometry, 119-136, Iwanami Shoten, Tokyo, 1977.

[Ta65] J. Tate, Algebraic cycles and poles of zeta functions, Arithmetic Algebraic Geometry, Harper and Row, 93-110 (1965).

[Ve13] M. Verbitsky, Degenerate twistor spaces for hyperkähler manifolds, arXiv:1311.5073 (2013).

[Zi84] T. Zink, Cartiertheorie kommutativer formaler Gruppen, Teubner (1984).

TU MÜnChen, Zentrum Mathematik - M11, BoltzMannstr. 3, D-85748 Garching BEI MÜNCHEN, GERMANY

Email address: liedtke@ma.tum.de 